\title{
Metallic Interface Emerging at Magnetic Domain Wall of Antiferromagnetic Insulator: Fate of Extinct Weyl Electrons
}

\begin{abstract}
Youhei Yamaji* and Masatoshi Imada ${ }^{\dagger}$
Department of Applied Physics, University of Tokyo, Hongo, Bunkyo-ku, Tokyo 113-8656, Japan

(Received 15 July 2013; revised manuscript received 3 November 2013; published 27 May 2014)

Topological insulators, in contrast to ordinary semiconductors, accompany protected metallic surfaces described by Dirac-type fermions. Here, we theoretically show that another emergent two-dimensional metal embedded in the bulk insulator is realized at a magnetic domain wall. The domain wall has long been studied as an ingredient of both old-fashioned and leading-edge spintronics. The domain wall here, as an interface of seemingly trivial antiferromagnetic insulators, emergently realizes a functional interface preserved by zero modes with robust two-dimensional Fermi surfaces, where pyrochlore iridium oxides proposed to host the condensed-matter realization of Weyl fermions offer such examples at low temperatures. The existence of in-gap states that are pinned at domain walls, theoretically resembling spin or charge solitons in polyacetylene, and protected as the edges of hidden one-dimensional weak Chern insulators characterized by a zero-dimensional class-A topological invariant, solves experimental puzzles observed in $R_{2} \mathrm{Ir}_{2} \mathrm{O}_{7}$ with rare-earth elements $R$. The domain wall realizes a novel quantum confinement of electrons and embosses a net uniform magnetization that enables magnetic control of electronic interface transports beyond the semiconductor paradigm.
\end{abstract}

DOI: 10.1103/PhysRevX.4.021035

\section{INTRODUCTION}

Interfaces in semiconductor heterojunctions, field-effect transistors, and between vacuum and newly characterized topologically nontrivial semiconductors host various twodimensional electron systems tightly confined around these interfaces, which offer major playgrounds of electronics and spintronics. Especially, topologically nontrivial semiconductors classified as topological insulators or Chern insulators [1,2], in contrast to the usual band insulators, accompany protected metallic surfaces described by Dirac-type fermions [3-5]. Another peculiar metallic state with a truncated Fermi surface called an "arc" is predicted [6] on the interfaces between vacuum and a newly recognized class of zero-gap semiconductor [7,8]: It hosts a condensed-matter realization of Weyl fermions, initially proposed in iridium pyrochlore oxides $R_{2} \mathrm{Ir}_{2} \mathrm{O}_{7}$ ( $R=$ rare-earth elements) under a magnetic order [9-14].

In this article, we unveil that magnetic domain walls offer qualitatively novel interfaces in magnetically ordered zero-gap semiconductors such as $R_{2} \mathrm{Ir}_{2} \mathrm{O}_{7}$, which are

\footnotetext{
*yamaji@solis.t.u-tokyo.ac.jp

imada@ap.t.u-tokyo.ac.jp
}

Published by the American Physical Society under the terms of the Creative Commons Attribution 3.0 License. Further distribution of this work must maintain attribution to the author(s) and the published article's title, journal citation, and DOI.
Subject Areas: Condensed Matter Physics,

Strongly Correlated Materials,

Topological Insulators

expected to host Weyl fermions in the bulk. Magnetic domain walls have historically been of interest in both fundamental physics [15] and technology [16] as an archetypical and fundamental model for inhomogeneity originating from spontaneous symmetry breaking and, for example, as an essential ingredient for antique magneticbubble memory. Recently, applications of spintronics, such as magnetic random-access memories, have received renewed interest in electric controls of magnetic domain walls. We theoretically show, differently from these aspects and applications of magnetic domain walls, that a class of magnetic domain walls induces unexpected interface metals accompanied by a net uniform magnetization, brought about by the insertion of the domain wall, in the background of a seemingly trivial bulk antiferromagnetic insulator, where uniform magnetization is canceled out in the bulk. The metallicity of the domain wall is triggered by the formation of Fermi arcs at the domain walls, which originate from the condensed-matter Weyl fermions, or the Weyl electrons, while the Fermi arc evolves into the Fermi surface when the Weyl fermions are eliminated as detailed in this article.

Robustness against perturbations and the anomalous electromagnetic responses of Weyl fermions arising from the chiral anomaly are the reasons why the condensedmatter realization of Weyl fermions has been interesting experimentally and theoretically [17]. The Weyl electrons are, however, easily annihilated in a pair with Weyl 
electrons of the opposite chirality. Consequently, the Fermi arc on the surface of the pyrochlore zero-gap semiconductor survives only near the all-in-all-out-type antiferromagnetic transition temperature $[12,18]$.

In contrast to fragile Fermi arcs at surfaces, here, we show that magnetic domain walls realize metallic interfaces preserved by zero modes or in-gap states with a robust Fermi arc or Fermi surface even after the pair annihilation of Weyl electrons and even in the seemingly trivial antiferromagnetic insulators, as summarized in Fig. 1. The zero modes follow a one-dimensional Dirac equation that protects in-gap states. The existence of a gapless mode at the domain wall is protected because the bulk state is projected to a one-dimensional weak Chern insulator, provided that certain symmetries are satisfied. The domain wall can indeed satisfy these symmetries. Namely, persisting metallicity pinned at domain walls is assumed. The domain walls also maintain a ferromagnetic moment, similarly to spin solitons in polyacetylene. It may solve experimental puzzles of the iridium oxides, such as bad insulating behaviors $[9,10]$ with clear optical gaps [12], anomalous weak ferromagnetism [10,13], and anomalous magnetotransports $[14,19]$ widely observed in the pyrochlore iridium oxides, regardless of their detailed chemical compositions. Furthermore, it offers a novel quantum confinement of electrons, enabling magnetic control of interface electronic transports. For quantum wells or crystal grain boundaries, the location, orientation, and number of these interfaces cannot be controlled after their fabrications. In contrast, our magnetic domain walls are tunable through applied magnetic fields and further host protected in-gap metallic domain-wall states.

Dirac- or Weyl-type fermions [20,21] realized in crystalline solids with both strong spin-orbit couplings and Coulomb repulsion are a subject of intensive studies [7,18,22-24]. We elucidate another prominent effect arising from the combined interaction and topology by studying a single-band Hubbard-type model on the pyrochlore lattice [Fig. 2(a)], with the $J_{\text {eff }}=1 / 2$ manifold of the iridium pyrochlore oxides in mind, where $J_{\text {eff }}$ is an effective total angular momentum of the $5 d$ orbitals of an iridium atom with five electrons: We show that the Weyl electrons leave behind their indelible trace with a Fermi surface at the magnetic domain walls even after the pair annihilations of them, namely, even when the Weyl electrons completely disappear and the bulk and surface turn into an insulator. This conclusion is supported by fully unrestricted Hartree-Fock calculations, where the

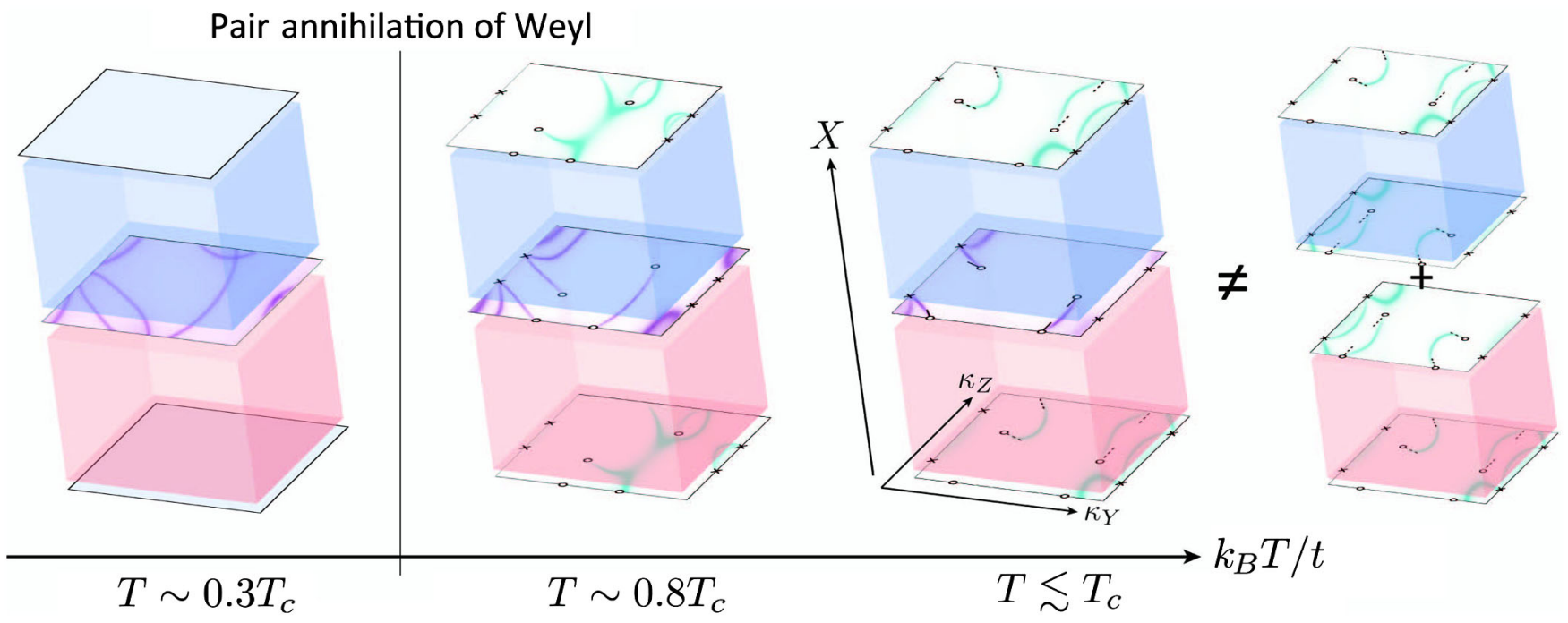

FIG. 1. Two-dimensional Brillouin zones illustrated for two different kinds of interfaces, namely, surfaces of bulk crystals with magnetic orders (red or blue transparent cubes) against vacua and domain walls between two magnetic domains. Evolution of Fermi arcs is shown for the surfaces between the bulk and vacua and for the magnetic domain walls between two magnetic domains upon changes in temperatures $T$ (indicated by the horizontal axis). The evolution occurs in a zero-gap semiconductor hosting Weyl electrons below its critical temperatures for the magnetic order $T_{c}$. The domains with two inequivalent magnetic orders that are mapped onto each other through the time-reversal operation are illustrated as the red and blue transparent cubes (called "all-out" and "all-in" domains in the later discussion). These surfaces and domain walls are illustrated to be perpendicular to the $X$ axis. The spectral functions at the Fermi level are shown in the momentum frame $\left(\kappa_{Y}, \kappa_{Z}\right)$ with a finite broadening factor for the sake of illustration. Here, stronger colors indicates higher spectral intensities. In contrast to the naive expectation that the magnetic domain walls simply consist of two surfaces between the bulk and vacua (shown in the right end), the magnetic domain wall offers a novel two-dimensional interface distinct from the surfaces: Even after pair annihilation of the Weyl electrons, the domain-wall metallic states remain and form a Fermi surface (shown in the left end panel). All of the Fermi arcs (and the Fermi surface at lowest temperatures) are calculated by using the tight-binding Hamiltonian (1) with the $(01 \overline{1})$ surfaces and domain walls introduced later, with the parameter set used for Figs. 3 and 6 . For detailed notations, see the captions of Figs. 3 and 6. 
self-consistent mean fields at every atom for charge density and three spin components are fully relaxed, as well as by analyses on the Dirac equations for the effective lowenergy model. Electronic states bound around the domain walls are formed, whose origins are traced back to the bulk Weyl electrons and their quantum chiral anomaly. The present domain-wall theory offers insights into a number of peculiar properties of $R_{2} \mathrm{Ir}_{2} \mathrm{O}_{7}$, including weak ferromagnetism with strong field dependence [13] bad but stubborn electronic conduction [12] and negative magnetoresistance [14].

The outline of the present paper is as follows: A theoretical model for pyrochlore iridium oxides is defined in Sec. II. In Sec. III, Weyl electrons and their pair annihilation in the present model are described. We employ the standard $\vec{k} \cdot \vec{p}$ perturbation theory around the Fermi level, which generates the Luttinger Hamiltonian, as is derived in Appendixes A and B. In Sec. IV, together with Appendix D, the Luttinger Hamiltonian is reduced to onedimensional Dirac or three-dimensional Weyl Hamiltonians characterizing low-energy electronic states around magnetic domain walls (defined in Appendix C) that describe the bulk Weyl electrons and Fermi arcs or surfaces appearing at the domain walls. Topological properties of the domain-wall states are elucidated in Sec. V and Appendix E as well as in a simplified model in Sec. VI. The similarity of the domain wall to the solitons in polyacetylene is discussed. Unrestricted Hartree-Fock results on the model are given in Sec. VII. It is shown that the one-dimensional Dirac equation captures the essence of the full effective tightbinding model for the pyrochlore iridium oxides. Symmetric properties and the symmetry-protected topological nature of the domain-wall states are detailed in Sec. VIII. The relevance of our theoretical predictions to experimental observations on pyrochlore iridium oxides is discussed in Sec. IX. Bad insulating properties in the electronic conduction and weak ferromagnetism, as well as large magnetoresistance in $\mathrm{Nd}$ and $\mathrm{Gd}$ compounds observed experimentally, are naturally understood from the present theory.

\section{MODEL OF PYROCHLORE IRIDIUM OXIDES WITH SPIN-ORBIT INTERACTION}

In this article, we employ a simple model describing the essential physics of iridium pyrochlore oxides, which is also one of the minimal models hosting bulk Weyl fermions: The Hubbard Hamiltonian with the on-site interaction $U$, transfer $t$, and spin-orbit coupling $\zeta$ decoded as spin-dependent imaginary hopping at the filling of one electron per site is introduced as

$$
\begin{aligned}
\hat{H}= & -t \sum_{i, j}^{\mathrm{NN}} \sum_{\sigma}\left[\hat{c}_{i \sigma}^{\dagger} \hat{c}_{j \sigma}+\text { H.c. }\right]+U \sum_{i} \hat{n}_{i \uparrow} \hat{n}_{i \downarrow} \\
& +i \zeta \sum_{i, j}^{\mathrm{NN}} \sum_{\alpha, \beta=\uparrow, \downarrow} \hat{c}_{i \alpha}^{\dagger}\left(\overrightarrow{\hat{\sigma}} \cdot \frac{\vec{b}_{i j} \times \vec{d}_{i j}}{\left|\vec{b}_{i j} \times \vec{d}_{i j}\right|}\right)_{\alpha \beta} \hat{c}_{j \beta},
\end{aligned}
$$
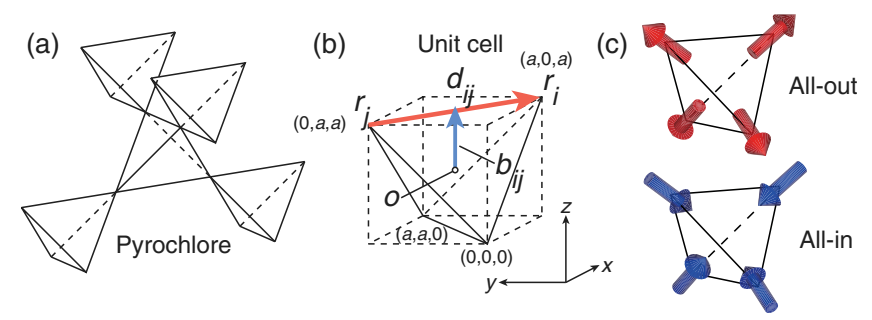

FIG. 2. Magnetic structure and notations for spin-orbit interaction on pyrochlore lattice. (a) Pyrochlore lattice structure, (b) definition of effective spin-orbit couplings on pyrochlore lattices, and (c) all-in-all-out magnetic-moment configuration. In (b), the vectors $\vec{d}_{i j}$ and $\vec{b}_{i j}$ are illustrated for a specific bond. The vector $\vec{d}_{i j}$ points from the $j$ th site $\vec{r}_{j}$ to the $i$ th site $\vec{r}_{i}$, and the vector $b_{i j}$ points from the center of the unit tetrahedron $O$ to the midpoint between $\vec{r}_{i}$ and $\vec{r}_{j}$.

where a fermionic operator $\hat{c}_{i \sigma}^{\dagger}\left(\hat{c}_{i \sigma}\right)$ creates (annihilates) an electron with $\sigma$ spin at the $i$ th site. Here, the effective spinorbit coupling described by the $\zeta$ term is given by pseudovectors $\vec{b}_{i j} \times \vec{d}_{i j}$ illustrated in Fig. 2(b). This $\zeta$ term is the unique form of the spin-orbit interaction as the nearest-neighbor (NN) hopping matrices allowed by the time-reversal symmetry and the point-group symmetry of the pyrochlore lattice, except for rotations of the global spin-quantization axis.

The sign of $\zeta$ determines the electronic structure of the model $[25,26]$ and its ground-state magnetism: The zerogap semiconductors are realized for $\zeta<0$, while, for $\zeta>0$, the system becomes a topological insulator in the absence of interaction. The magnetic ground state for $\zeta<0$ and $U>0$ is the all-in-all-out order [Fig. 2(c)], where the magnetic moment at each site points away from or toward the center of the tetrahedron and feels like Ising-type anisotropy [27]. Thus, there remains twofold degeneracy of the order.

\section{WEYL ELECTRONS AND THEIR ANNIHILATION IN PAIRS}

Once the all-in-all-out orders are formed, the physics of Weyl electrons [the low-energy physics of the Hamiltonian (1) with $\zeta<0$ ] is essentially captured by mean-field decouplings of the short-ranged Coulomb repulsion $U$, except for quantitative corrections arising from gapped quantum and/or thermal spin fluctuations and irrelevant quasiparticle renormalizations.

If the Coulomb repulsion $U$ is neglected, the low-energy electronic energy-momentum dispersion is described by a variation of the Luttinger Hamiltonian [22,28,29], which is a prototypical four-component effective Hamiltonian for semiconductors with the cubic symmetry. The explicit form of the effective Hamiltonian directly derived from Eq. (1) is given as 


$$
\hat{h}_{4 \times 4}(\vec{k})=\left[+2 t\left(1-\frac{k^{2}}{3}\right)-2 \sqrt{2}|\zeta|\right] \mathbf{1}_{4}-2 t \vec{d}(\vec{k}) \cdot \overrightarrow{\hat{\Gamma}}
$$

where

$$
\vec{d}(\vec{k})^{T}=-\left(\frac{k_{y} k_{z}}{\sqrt{3}}, \frac{k_{z} k_{x}}{\sqrt{3}}, \frac{k_{x} k_{y}}{\sqrt{3}}, \frac{k_{x}^{2}-k_{y}^{2}}{2 \sqrt{3}}, \frac{3 k_{z}^{2}-k^{2}}{6}\right),
$$

and a vector of $4 \times 4$ Dirac matrices $\vec{\Gamma}^{T}=\left(\hat{\Gamma}^{1}, \hat{\Gamma}^{2}\right.$, $\left.\hat{\Gamma}^{3}, \hat{\Gamma}^{4}, \hat{\Gamma}^{5}\right)$ is defined as

$$
\begin{aligned}
& \hat{\Gamma}^{1}=\left[\begin{array}{cc}
0 & -i \hat{\sigma}_{0} \\
+i \hat{\sigma}_{0} & 0
\end{array}\right], \\
& \hat{\Gamma}^{2}=\left[\begin{array}{cc}
0 & +\hat{\sigma}_{z} \\
+\hat{\sigma}_{z} & 0
\end{array}\right], \\
& \hat{\Gamma}^{3}=\left[\begin{array}{cc}
0 & +\hat{\sigma}_{y} \\
+\hat{\sigma}_{y} & 0
\end{array}\right], \\
& \hat{\Gamma}^{4}=\left[\begin{array}{cc}
0 & +\hat{\sigma}_{x} \\
+\hat{\sigma}_{x} & 0
\end{array}\right], \\
& \hat{\Gamma}^{5}=\left[\begin{array}{cc}
+\hat{\sigma}_{0} & 0 \\
0 & -\hat{\sigma}_{0}
\end{array}\right] .
\end{aligned}
$$

(See Appendix A for the derivation.) The low-energy bands are degenerate quadruply at the crystallographic $\Gamma$ point, the center of the Brillouin zone in the momentum space $\vec{k}=(0,0,0)$, and form a so-called quadratic band crossing.

By adding a small but finite mean-field term representing the all-in-all-out orders $m \hat{\Gamma}^{54}$ with another Dirac matrix $\hat{\Gamma}^{54}=\left[\hat{\Gamma}^{5}, \hat{\Gamma}^{4}\right] / 2 i$ (see Appendix A for the microscopic derivation) and $m=U m_{\text {all }} / 2$, eight Weyl points at the momenta $\vec{k}=\vec{k}_{\mathrm{Weyl}} \simeq \sqrt{|m| / 2 t}( \pm 1, \pm 1, \pm 1)$, up to the order of $|m|$, are induced instead, while the fourfold degeneracy at the $\Gamma$ point is lifted. Here, $m_{\text {all }}$ represents the amplitude of the magnetic moment at each site in the all-in-all-out phase. The energy spectrum is given through the poles of the Green's function

$$
\hat{G}_{4 \times 4}(\vec{k}, \omega)=\left[(\omega+\mu) \mathbf{1}_{4}-\hat{h}_{4 \times 4}(\vec{k})\right]^{-1},
$$

as

$$
E(\vec{k})=-\mu \pm \sqrt{4 t^{2}|\vec{d}(\vec{k})|^{2}+m^{2} \pm 4|m| t \sqrt{d_{1}(\vec{k})^{2}+d_{2}(\vec{k})^{2}+d_{3}(\vec{k})^{2}}}
$$

where $\mu$ is the chemical potential. The momenta of the Weyl points are given by the equations $d_{4}(\vec{k})=d_{5}(\vec{k})=0$ and $2 t|\vec{d}(\vec{k})|=|m|$. (See Appendix B for more details.) When the order parameter $m$ increases and becomes comparable to $t$, these eight Weyl points come closer and are annihilated in pairs at the crystallographic $L$ points $\vec{k}=\vec{k}_{L}=( \pm \pi / 4 a, \pm \pi / 4 a, \pm \pi / 4 a)$ at the boundary of the Brillouin zone. We note that there are only four inequivalent $L$ points.

To understand the nature of bulk Weyl electrons, their pair annihilation, Fermi arcs on surfaces, and ones on domain walls, it is sufficient to employ a $\vec{k} \cdot \vec{p}$ perturbation theory, which is a traditional technique for a semiconductor and its interface physics [30] around the Weyl points, starting from the four-component effective Hamiltonian $\hat{h}_{4 \times 4}(\vec{k})+m \hat{\Gamma}^{54}$. Especially, near the $\Gamma$ point and the $L$ points, the $\vec{k} \cdot \vec{p}$ perturbation theory gives us simple expressions suitable for the exploration of the physics of interfaces, as shown in Sec. IV. As unperturbed wave functions for the $\vec{k} \cdot \vec{p}$ perturbation theory, we choose the wave functions at these symmetric points classified by irreducible representations of the point groups $T_{d}$ and $D_{3 d}$ for the $\Gamma$ point and the $L$ points, respectively. Around the $\Gamma$ point, the $\vec{k} \cdot \vec{p}$ Hamiltonian expanded from the quartet labeled by $G_{3 / 2}$ in the terminology of the point group $T_{d}$ is nothing but the Luttinger Hamiltonian discussed above. The four-component Hilbert space labeled by irreducible representations $E_{3 / 2 u} \oplus E_{1 / 2 g}$ at the $L$ point gives the basis for the $\vec{k} \cdot \vec{p}$ Hamiltonian around the $L$ points. Once the $\vec{k} \cdot \vec{p}$ perturbation theory around the Weyl points near the $\Gamma$ point and $L$ points is obtained, it leads to the entire description of the Weyl electrons through the interpolation of these low-energy theories around the $\Gamma$ point and the $L$ points.

Here, we elucidate the relevance of our model hosting the eight Weyl points to the pyrochlore iridium oxides $R_{2} \mathrm{Ir}_{2} \mathrm{O}_{7}$. First of all, the quadratic band crossing and fourfold degeneracy at the $\Gamma$ point are symmetry-protected properties of the $J_{\text {eff }}=1 / 2$ manifold on the pyrochlore lattice. When the time-reversal symmetry is broken with keeping the $T_{d}$ symmetry, the eight Weyl points immediately stem from the $\Gamma$ point. When the system becomes insulating, the pair annihilation of the eight Weyl points necessarily occurs through the level crossing at the $L$ points between the Zeeman-split states from the $E_{1 / 2 g}$ and $E_{3 / 2 u}$ doublets discussed in the paragraph above.

On the other hand, 24 Weyl points are found by the calculation based on the local spin density approximation (LSDA) supplemented by taking account of the spin orbit (SO) interaction and the electron correlation effect on the 
Hartree-Fock level, namely, so-called LSDA $+\mathrm{SO}+U$ calculation in Ref. [6]. The 24 Weyl points are created as three pairs at each of four $L$ points when the split states from the $E_{1 / 2 g}$ and $E_{1 / 2 u}$ doublets at the $L$ points show level crossings, instead of states from the $E_{1 / 2 g}$ and $E_{3 / 2 u}$ doublets. These 24 Weyl points are nothing to do with the eight Weyl points discussed in this paper. As detailed in the following section, what we find is that the eight Weyl points leave gapless domain-wall states as a topological nature of pyrochlore iridium oxides even after their pair annihilation, while the 24 Weyl points leave nothing. Furthermore, whether the crossing of the $E_{1 / 2 g}$ and $E_{1 / 2 u}$ doublets occurs depends on the details of the material parameters and does not always happen. The eight Weyl points are not focused on in Ref. [6], although signs of the eight Weyl points are found inside the semimetal phase with Fermi surfaces in the band dispersion.

\section{EFFECTIVE ONE-DIMENSIONAL DIRAC HAMILTONIAN}

Under the influence of a small but finite order parameter $m$, the four-component effective Hamiltonian $\hat{h}_{4 \times 4}(\vec{k})+$ $m \hat{\Gamma}^{54}$ exhibits Weyl points consisting of two of the four components, while the other two components are gapped. [See Eq. (5) and Appendix D.] Around the Weyl points, the Hamiltonian therefore breaks up into a pair of twocomponent Hamiltonians, up to the linear order in the $\vec{k} \cdot \vec{p}$ perturbation, one of which is nothing but a Weyl Hamiltonian describing three-dimensional massless fermions. For $m>0$, we note the Weyl Hamiltonian as $\hat{h}_{\Gamma \vec{k}_{\text {Weyl }}}^{(+)}\left(\hat{h}_{L \vec{k}}^{(+)}\right)$and describe the other two-component gapped part as $\hat{h}_{\Gamma \vec{k}_{\text {weyl }}}^{(-)}\left(\hat{h}_{L \vec{k}}^{(-)}\right)$, around the $\Gamma$ point (the $L$ points). For instance, the four-component effective Hamiltonian is expanded with respect to the momentum measured from the Weyl points $\delta \vec{k}$ as

$$
\begin{aligned}
& \hat{h}_{4 \times 4}\left(\vec{k}_{\text {Weyl }}+\delta \vec{k}\right)+m \hat{\Gamma}^{54} \rightarrow \\
& {\left[\begin{array}{cc}
\hat{h}_{\Gamma \vec{k}_{\text {Weyl }}}^{(+)}(\delta \vec{k}) & \mathcal{O}(t|\delta \vec{k}|) \\
\mathcal{O}(t|\delta \vec{k}|) & \hat{h}_{\Gamma \vec{k}_{\text {Weyl }}^{(-)}}^{(\delta \vec{k})}
\end{array}\right]+\mathcal{O}\left(t^{2}|\delta \vec{k}|^{2}\right),}
\end{aligned}
$$

around the $\Gamma$ point, after an appropriate unitary transformation independent of $m$. Since the gapped part

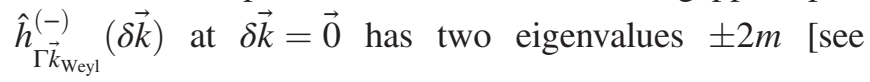
Eq. (5)] with $m>0$, the low-energy excitations up to $t|\overrightarrow{\delta k}|$ are described by the Weyl Hamiltonian $\hat{h}_{\Gamma_{k_{\text {weyl }}}^{(+)}}$at a small $\delta \vec{k} \neq \overrightarrow{0}$. Surprisingly, when we change the sign of $m$ while keeping its amplitude, or simply apply the timereversal operator to the Hamiltonian, the role interchanges and the two-component Hamiltonian $\hat{h}_{\Gamma \vec{k}_{\text {weyl }}}^{(-)}\left(\hat{h}_{L \vec{k}}^{(-)}\right)$ describes Weyl electrons, while $\hat{h}_{\Gamma \vec{K}_{\text {Weyl }}}^{(+)}\left(\hat{h}_{L \vec{k}}^{(+)}\right)$describes gapped components.

As elucidated in the literature [6,31,32], bulk Weyl electrons result in Fermi arcs on surfaces and/or domain walls of the bulk crystals. By using the $\vec{k} \cdot \vec{p}$ Hamiltonians $\hat{h}_{\Gamma \vec{k}_{\text {Weyl }}}^{( \pm)}$or $\hat{h}_{L \vec{k}}^{( \pm)}$around the Weyl points, we can sketch the Fermi arcs not only on the surfaces but also on the magnetic domain walls in the following.

For clarification, we concentrate on a pair of Weyl points $\vec{k}_{\text {Weyl }}= \pm \sqrt{|m| / 2 t}(1,1,1)$, with $|m| / t \ll 1$, and on a surface or domain wall perpendicular to $(0,+1,-1)$, namely, a $(01 \overline{1})$ surface or domain wall. In the following discussion, we call a coordination axis along $(0,+1,-1)$ the $X$ axis and introduce an oblique coordinate $(X, Y, Z)$ together with the corresponding reciprocal momentum coordinate $\left(\kappa_{X}, \kappa_{Y}, \kappa_{Z}\right)$ defined through

$$
\vec{r}=X\left[\begin{array}{c}
0 \\
+2 a \\
-2 a
\end{array}\right]+Y\left[\begin{array}{c}
0 \\
-2 a \\
-2 a
\end{array}\right]+Z\left[\begin{array}{c}
-4 a \\
+2 a \\
+2 a
\end{array}\right]
$$

and

$$
\vec{k}=\kappa_{X}\left[\begin{array}{c}
0 \\
+1 / 4 a \\
-1 / 4 a
\end{array}\right]+\kappa_{Y}\left[\begin{array}{c}
-1 / 4 a \\
-1 / 4 a \\
-1 / 4 a
\end{array}\right]+\kappa_{Z}\left[\begin{array}{c}
-1 / 4 a \\
0 \\
0
\end{array}\right] .
$$

[See also Fig. 3(a) and Appendix C.] As detailed above, around the Weyl points, the Luttinger Hamiltonian breaks up into a pair of the following two-component Dirac Hamiltonians $\hat{h}_{\Gamma \vec{k}_{\text {weyl }}}^{(+)}$and $\hat{h}_{\Gamma \vec{k}_{\text {Weyl }}}^{(-)}$that describe low-energy physics in the all-out and all-in domains, with $m>0$ and $m<0$, respectively. (See Appendix D.) For $\vec{k}_{\mathrm{Weyl}}=$ $\kappa_{0}(1 / \sqrt{3}, 1 / \sqrt{3}, 1 / \sqrt{3})$, with $\kappa_{0}= \pm \sqrt{3|m| / 2 t}$, the twocomponent Dirac Hamiltonian up to the linear order in $-i \partial_{X}, \delta \kappa_{Y}$, and $\kappa_{Z}$ is given as

$$
\begin{aligned}
\hat{h}_{\Gamma \vec{k}_{\text {Weyl }}^{( \pm)}\left(-i \partial_{X}, \delta \kappa_{Y}, \kappa_{Z} ; X\right)=} & h_{0}\left(\delta \kappa_{Y}, \kappa_{Z}\right) \hat{\sigma}_{0} \\
& +h_{x}\left(\delta \kappa_{Y}, \kappa_{Z}\right) \hat{\sigma}_{x}+h_{y}\left(-i \partial_{X}\right) \hat{\sigma}_{y} \\
& +h_{z}^{( \pm)}\left(\delta \kappa_{Y}, \kappa_{Z}, X\right) \hat{\sigma}_{z}
\end{aligned}
$$

where we introduce a new variable $\delta \kappa_{Y}$ defined through $\left(\kappa_{X}, \kappa_{Y}=\kappa_{0} / \sqrt{3}+\delta \kappa_{Y}, \kappa_{Z}\right)$ and replace $\kappa_{X}$ with $-i \partial_{X}$. (See Appendix D for the derivation.) The Weyl points are projected to $\left(\delta \kappa_{Y}, \kappa_{Z}\right)=(0,0)$. Here, coefficients of the identity matrix and Pauli matrices in the Dirac Hamiltonian (9) are derived from the original Hamiltonian (1), via the low-energy Luttinger Hamiltonian, as 
(a)

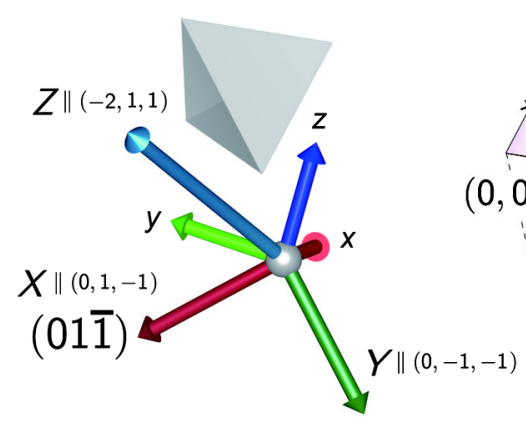

$(0,2 \pi)$ Domain wall

(b)

$(0,0)$

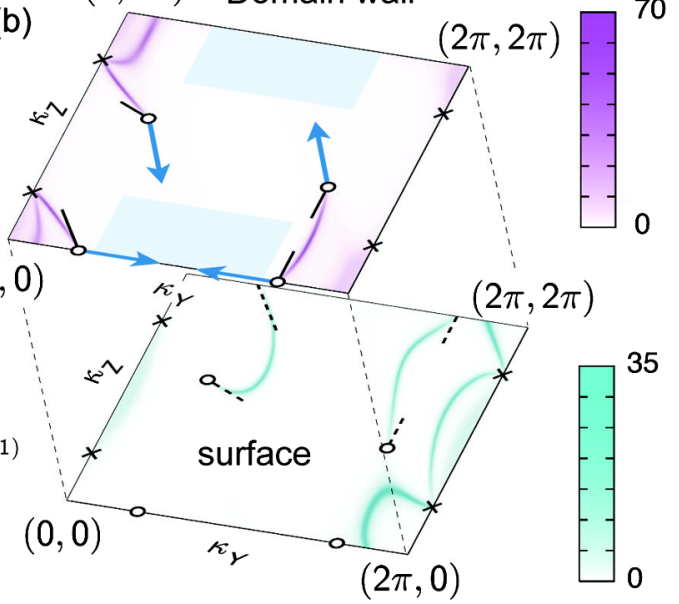

(c)

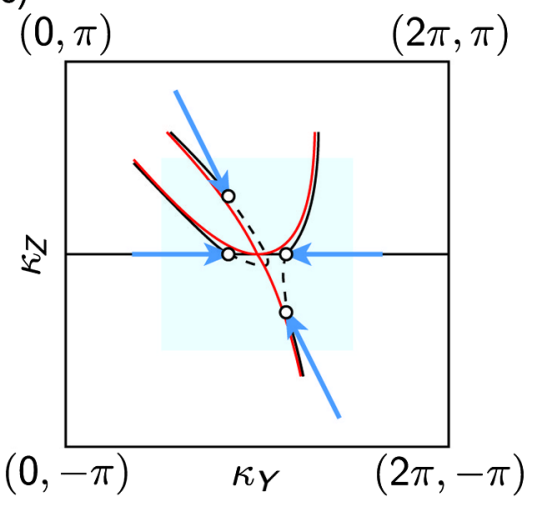

FIG. 3. Summary of solutions for effective one-dimensional Dirac equations $\hat{h}_{\Gamma \vec{k}_{\text {Weyl }}}^{( \pm)} \vec{\psi}(X)=E \vec{\psi}(X)$. (a) Newly introduced coordinate axes $X, Y$, and $Z$ are illustrated for the $(01 \overline{1})$ domain wall. (b) Zero modes of the chiral Dirac equations with low-energy $\vec{k} \cdot \vec{p}$ Hamiltonians including $\hat{h}_{\Gamma \kappa_{0} / \sqrt{3}(1,1,1)}^{( \pm)}$[Eq. (9)] and $\hat{h}_{\Gamma \kappa_{0} / \sqrt{3}(-1,1,1)}^{( \pm)}$(see Appendix D). Open circles indicate the four Weyl points projected to the $\left(\kappa_{Y}, \kappa_{Z}\right)$ plane at $\vec{k}_{\text {Weyl }}= \pm \sqrt{|m| / 2 t}(1,1,1)$ and $\vec{k}_{\text {Weyl }}= \pm \sqrt{|m| / 2 t}(-1,1,1)$. Crosses indicate the other four bulk Weyl points. Solid (dashed) lines represent the initial slopes of the loci of the domain-wall (surface) states starting from the Weyl points obtained from Eq. (2), where the full solution of Eq. (1) is illustrated by a color contour plot. Small deviations of the solid (dashed) black lines from the expectation from the contour plot may be ascribed to the small error arising from the reduction from the four-component Luttinger Hamiltonian to the two-component Hamiltonian (9), where off-diagonal elements in the order of $k^{2}$ are ignored. For $m \rightarrow 0$, solid curves shrink and disappear at $(0,0)$. Arrows indicate the directions along which the projected Weyl points move when $|m|$ increases. (c) Qualitative description for pair annihilation of bulk Weyl electrons on the $\left(\kappa_{Y}, \kappa_{Z}\right)$ plane. Solid (dashed) curves illustrate the loci of the domain-wall (surface) zero modes. When the projected Weyl points move along the direction indicated by the arrows originally starting from $(0,0)$, the pair annihilation occurs at $\left(\kappa_{Y}, \kappa_{Z}\right)=(\pi, 0)$. Then, the closed loop of the Fermi surface on the domain walls appears, which is represented by the solid red curves. Note that the Brillouin zone is shifted $(0, \pi)$ from (b). The shaded regions in (b) and (c) represent the same area.

$h_{0}=-4 \sqrt{3} t \kappa_{0}\left(\delta \kappa_{Y}+\kappa_{Z} / 3\right), h_{x}=4 \sqrt{3} t \kappa_{0} \kappa_{Z} / 3, h_{y}=4 t \kappa_{0} i \partial_{X}$, and $h_{z}^{( \pm)}=\mp 4 t \kappa_{0}\left(\delta \kappa_{Y}+\kappa_{Z} / 3\right) / \sqrt{3}+m(X) \mp|m|$.

Then, the two-component one-dimensional Dirac equation $\hat{h}_{\Gamma \vec{k}_{\text {Weyl }}}^{(+)} \vec{\psi}(X)=E \vec{\psi}(X)$ gives a description of bound states on the surface or domain walls by introducing suitable $X$-dependent "mass" terms $m(X)[33,34]$. Here, the all-out (all-in) domain is described by $m(X)=+|m|$ $[m(X)=-|m|]$. We also note that, if $|m|$ is large enough, the Weyl points are annihilated in pairs and the bulk system becomes a trivial magnetic insulator. Therefore, the mass term $m(X)=|m| \theta(-X)-|m| \theta(X)$ gives a description of the magnetic domain wall at $X=0$ for long-wavelength behaviors.

The $X$-dependent mass term for the magnetic domain walls introduced above indeed reproduces the numerical solution of the tight-binding Hamiltonian (1) for the Fermi arcs (solid curves) around the Weyl points (white circles) projected onto the domain-wall Brillouin zone, at least up to linear order, as shown in Fig. 3(b). It shows the validity of the effective one-dimensional Dirac equation for the domain-wall Fermi arcs.
Then, we explain how a description for a surface between a vacuum $(X<0)$ and the bulk $(X>0)$ can be mimicked by $m(X)=M \times \operatorname{sgn}(m) \theta(-X)+m \theta(X)$, with $M \gg|m|$. The introduction of the large amplitude of the mass $M$ without the sign change in $m(X)$ mimics a zeroFermi-velocity limit and indeed offers an effective description of vacuum. In the 1D Chern insulator, topologically trivial phases with the zero Chern number are realized by setting the Fermi velocity equal to 0 . By taking into account the fact that the relevant length scale governing the wave functions of the edge states is the ratio of the amplitude of the mass and the Fermi velocity, the small Fermi-velocity limit corresponds to the large mass-amplitude limit independently of the sign of the mass $M$. The comparison with the numerical solution of the tight-binding Hamiltonian (1) at the surface between the bulk and the real vacuum indeed supports the validity of the mass term $m(X)$, as shown in Fig. 3(b): The Fermi arcs (dashed curves) obtained with the mass term $m(X)$ around the projected Weyl points (white circles) are consistent with the numerical solution of Eq. (1), up to linear order.

When we treat three successive boundaries, such as ones between a vacuum and an all-out domain, between an 
all-out domain and an all-in domain, and between an all-in domain and a vacuum, we encounter a superficial problem with the choice of the mass term $m(X) \hat{\sigma}_{z}$ introduced above for a boundary between a vacuum and bulk: The vacua at both ends of the system have a different sign of $M$ and, therefore, are not seemingly connected to each other. In other words, there seem to exist two different vacua, which seems to be unphysical. However, if we take an atomic limit only inside the vacua described by the mass term, we can rotate the mass term and can connect these two vacua by applying unitary transformations such as $e^{i \pi \hat{\sigma}_{x} / 2}$ and changing the sign of $M$. We also remind the readers that the edgestate wave functions change smoothly when the atomic limits are taken [see concrete examples of the wave functions such as Eq. (D28)], while the vacua described by the mass term do not show the chiral anomaly [35] at the atomic limit.

As the order parameter $m$ develops, the two Weyl points at $\vec{k}=+\left|\kappa_{0}\right|(1 / \sqrt{3}, 1 / \sqrt{3}, 1 / \sqrt{3})$ and $\vec{k}=-\left|\kappa_{0}\right|(1 / \sqrt{3}$, $1 / \sqrt{3}, 1 / \sqrt{3})$ come closer and, finally, are annihilated in pairs at an $L$ point $\vec{k}_{L}=(\pi / 4 a, \pi / 4 a, \pi / 4 a)$. Around the $L$ point, the pair of the two-component Dirac Hamiltonian is given as

$$
\hat{h}_{L \vec{k}_{L}}^{( \pm)}=h_{x}\left(\kappa_{Z}\right) \hat{\sigma}_{x}+h_{y}\left(-i \partial_{X}, \kappa_{Z}\right) \hat{\sigma}_{y}+h_{z}^{( \pm)}(X) \hat{\sigma}_{z},
$$

where the coefficients of the Pauli matrices $h_{x}$ and $h_{y}$ are linear functions of their arguments, and $h_{z}^{( \pm)}=-m(X) \pm$ $|m|$. The above Dirac Hamiltonians (10) do not contain linear terms of $\delta \kappa_{Y}$, where $\left(\kappa_{Y}, \kappa_{Z}\right)=\left(\pi+\delta \kappa_{Y}, \kappa_{Z}\right)$, and, thus, the pair-annihilation point is given by $\left(\delta \kappa_{Y}, \kappa_{Z}\right)=$ $(0,0)$. Therefore, with a condition $\kappa_{Z}=0$ or $h_{x}=0$, the Dirac Hamiltonian (10) possesses chiral symmetry with a chiral operator $\hat{\sigma}_{x}$. (See Appendix E for the derivation and topological properties of the Dirac equation.)

Figure 3(b) illustrates an example of how the domainwall (solid curves) and surface (dashed curves) states extend around the Weyl points (white circles) before the pair annihilation at $\left(\kappa_{Y}, \kappa_{Z}\right)=(\pi, 0)$, shown in Fig. 3(c).

\section{CHIRAL ANOMALY}

In addition to the two-component 1D Dirac equations described above, the quantum chiral anomaly originating from the bulk Weyl nodes [35] also confirms the emergence of the domain-wall states. Below, we explain that the chiral anomaly due to bulk Weyl nodes leaves its trace even after the pair annihilation of the Weyl nodes, which inevitably induces domain-wall states.

Following Nielsen and Ninomiya [35], we start with Weyl fermions coupled to an external magnetic filed. Pairwise annihilation of two Weyl nodes with opposite chiralities coupled to an external magnetic field $(0,0, B)$ [or a vector potential $\left.\left(0, B r_{1}, 0\right)\right]$ is modeled by the following three-dimensional Weyl equation:

$$
\left[-i \partial_{r_{1}} \hat{\sigma}_{x}+\left(p_{2}-e B r_{1}\right) \hat{\sigma}_{y}+f\left(p_{3}\right) \hat{\sigma}_{z}\right] \psi=E \psi,
$$

where we introduce a real-space Cartesian coordinate $\left(r_{1}, r_{2}, r_{3}\right)$ and a corresponding momentum coordinate $\left(p_{1}, p_{2}, p_{3}\right)$, which are connected through $p_{a} \leftrightarrow-i \partial_{r_{a}}$ $(a=1,2,3)$. The microscopic origin of the above 3D Weyl equation is explained in Appendix E [see Eq. (E4) and the following paragraphs], although the 3D Weyl equation is a general one that describes the pairwise annihilation of Weyl nodes. The function depending on the third momentum coordinate $f\left(p_{3}\right)$ determines the chirality of the Weyl nodes and includes a mass term controlling the pairwise annihilation. For example, if we concentrate on the pair annihilation at an $L$ point $(\pi / 4 a, \pi / 4 a, \pi / 4 a)$ in the all-out domain with $m>0$, we can choose the function as $f\left(p_{3}\right)=+p_{3}^{2}-\left|m_{c}\right|+m$, where $\left|m_{c}\right|$ is a critical amplitude of the all-in-all-out magnetic moment for the pair annihilation. Here, the pairannihilation point, namely, the $L$ point, is represented by $p_{3}=0$ in the newly introduced momentum coordinate. The above Weyl equation leads to the following set of eigenvalues describing the Landau levels $E_{0}=\operatorname{sgn}(B) f\left(p_{3}\right)$ and $E_{n}=\operatorname{sgn}(n) \sqrt{f\left(p_{3}\right)^{2}+2 e|B||n|}$, where $n$ is a nonzero integer as $n= \pm 1, \pm 2, \pm 3, \ldots$. The emergence of the Landau level $E_{0}$ is nothing but a manifestation of the chiral anomaly.

Then, let us go into a detailed description of the domain wall based on the bulk Weyl equation introduced above. As already discussed, the low-energy physics of the all-in-allout phases is described by a pair of two-component Dirac equations: For a description on domain walls, we have introduced the 1D Dirac Hamiltonians $\hat{h}_{L \vec{k}_{L}}^{( \pm)}$. Inside an allout domain with $0<m\left(<\left|m_{c}\right|\right), \hat{h}_{L \vec{k}_{L}}^{(+)}$describes gapless excitations, while $\hat{h}_{L \vec{k}_{L}}^{(-)}$describes gapped excitations. On the other hand, inside an all-in domain with $0>m\left(>-\left|m_{c}\right|\right)$, $\hat{h}_{L \vec{k}_{L}}^{(-)}$corresponds to gapless ones, while $\hat{h}_{L \vec{k}_{L}}^{(+)}$corresponds to gapped ones. (For its illustration, see a later discussion in Fig. 10.) For replacing the $1 \mathrm{D}$ Dirac equations by a bulk 3 D Weyl equation, we introduce a set of $f\left(p_{3}\right)$ 's as

$$
\begin{aligned}
f^{(+)}\left(p_{3}\right)= & \theta(m)\left[+p_{3}^{2}-\left|m_{c}\right|+m\right] \\
& +\theta(-m)\left[-\left|m_{c}\right|+m\right]
\end{aligned}
$$

and

$$
\begin{aligned}
f^{(-)}\left(p_{3}\right)= & \theta(m)\left[+\left|m_{c}\right|+m\right] \\
& +\theta(-m)\left[-p_{3}^{2}+\left|m_{c}\right|+m\right] .
\end{aligned}
$$

Then, we obtain Landau levels illustrated in Fig. 4 with typical parameter sets. Here, we note that, even after the pair annihilation with $|m|>\left|m_{c}\right|$, the zeroth Landau level 

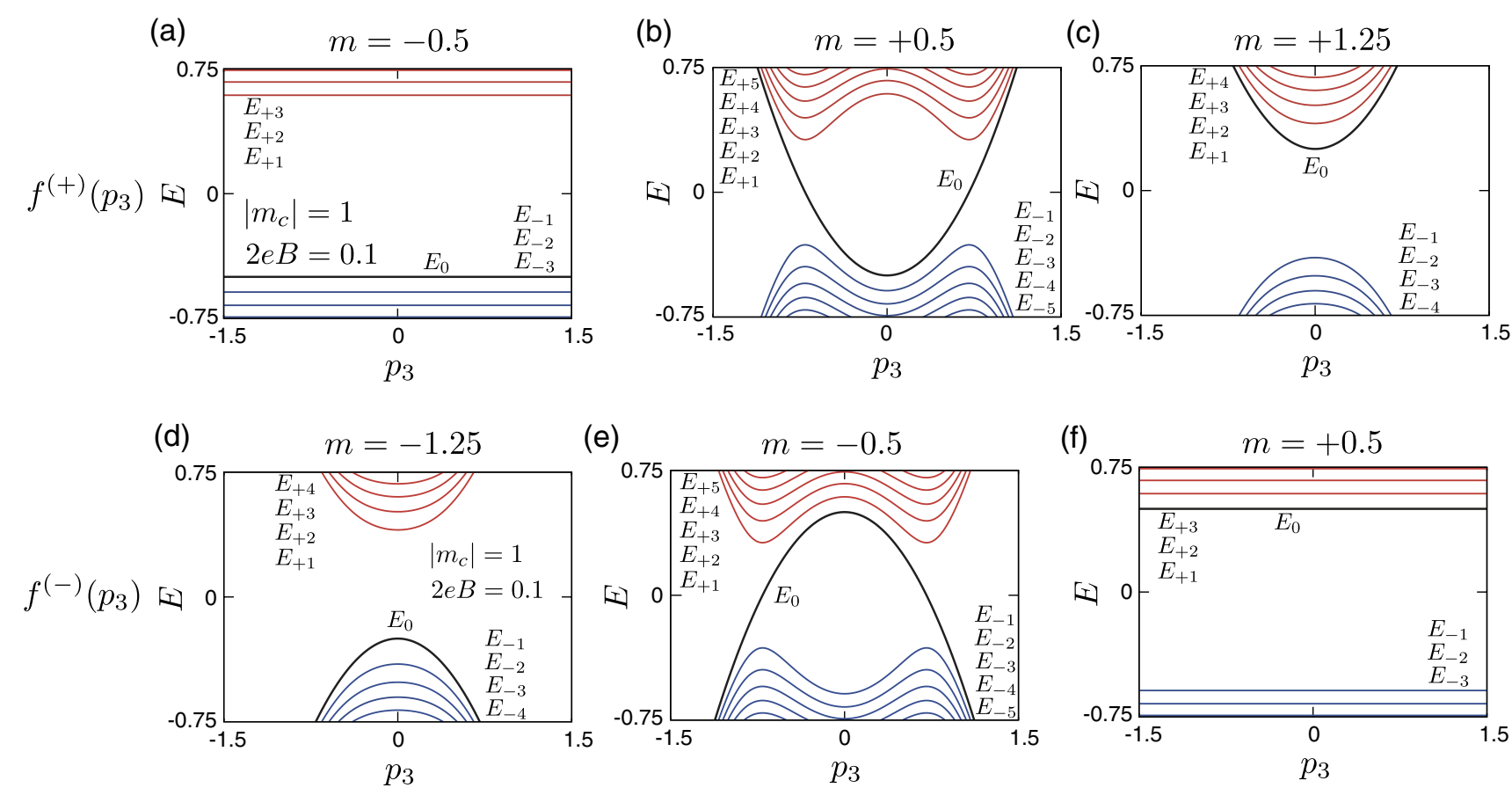

FIG. 4. Landau-level spectra for the 3D Weyl equation (11). Throughout this figure, we choose $\left|m_{c}\right|=1$ and $2 e B=0.1$. The Landau levels with $f^{(+)}\left(p_{3}\right)$ are shown for (a) $m=-0.5$, (b) $m=+0.5<\left|m_{c}\right|$, and (c) $m=1.25>\left|m_{c}\right|$. The Landau levels with $f^{(-)}\left(p_{3}\right)$ are also illustrated for (d) $m=-1.25<-\left|m_{c}\right|$, (e) $m=-0.5>-\left|m_{c}\right|$, and (f) $m=+0.5$. The Landau levels $E_{n}$ with $n>0$ ( $n<0$ ) are represented by solid red (blue) lines. The zeroth Landau levels $E_{0}$ are shown by solid black lines. For $m= \pm 0.5$, there are remnants of the Weyl nodes around $E=0$ : The zeroth Landau levels traverse $E=0$ from the valence bands to the conduction bands. The asymmetric nature of the zeroth Landau levels around $E=0$ is a manifestation of the chiral anomaly.

$E_{0}$ remains asymmetric, while other Landau levels $E_{n}$ are symmetric around the zero energy.

The structure of the Landau levels given by using Eqs. (11-13), illustrated in Fig. 4, directly leads to the emergence of the gapless domain-wall states. From the Landau-level spectrum in Fig. 4, the zeroth Landau levels for both $f^{(+)}\left(p_{3}\right)$ and $f^{(-)}\left(p_{3}\right)$ appear above $E=0$ and at the bottom of the conduction bands in the all-out domain with $m>0$. On the other hand, these zeroth Landau levels appear below $E=0$ and on top of the valence bands in the all-in domain with $m<0$. As a result, if the all-in and allout domains are smoothly connected to each other, these zeroth Landau levels are also smoothly connected and result in two gapless domain-wall states. Here, we remind the readers important facts that the two-dimensional Hilbert subspaces described by the 3D Weyl equations with $f^{( \pm)}\left(p_{3}\right)$ are orthogonal to each other, and the eigenvectors of the eigenvalues $E=E_{0}$ are orthogonal to those of the other Landau levels independently of the choices for $p_{3}, m$, and $e B$. We further detail in Sec. VIII the symmetry protection of the orthogonality by using the explicit symmetry satisfied by the domain walls.

Therefore, in addition to the topological nature of the 1D Dirac equations, the chiral anomaly as a bulk property protects the emergence of the gapless domain-wall states even after the pair annihilation of the bulk Weyl nodes. In both descriptions of the emergence of the domain-wall states, the domain-wall states are proven to appear in pairs. In contrast to the surface states of strong topological insulators, the degenerated or pairwise domain-wall states allow the occurrence of the Anderson localizations by the impurities that break the translational symmetry and degeneracy liftings due to additional spontaneous symmetry breakings. We note that similar domain-wall states are proposed in graphene with a broken inversion symmetry, characterized by asymptotic valley-resolved Chern numbers [36], and thus, by the parity anomaly.

\section{TOPOLOGICAL PROPERTIES OF DOMAIN- WALL STATES IN A SIMPLIFIED MODEL}

If the translational invariance along the domain walls is preserved, the in-gap states at these domain walls are protected by the chiral symmetry [2,37] of the Dirac Hamiltonian, particularly at a pair-annihilation point $\left(\kappa_{Y}, \kappa_{Z}\right)=(\pi, 0)$ (see Appendix E and also Sec. VIII for the protection by the symmetries) and by a generalized chiral symmetry [38] at other $k$ points. After the pair annihilation, only the loci of the domain walls survive.

The essential physics of these bound states is captured by the following toy model with higher symmetry, namely, 


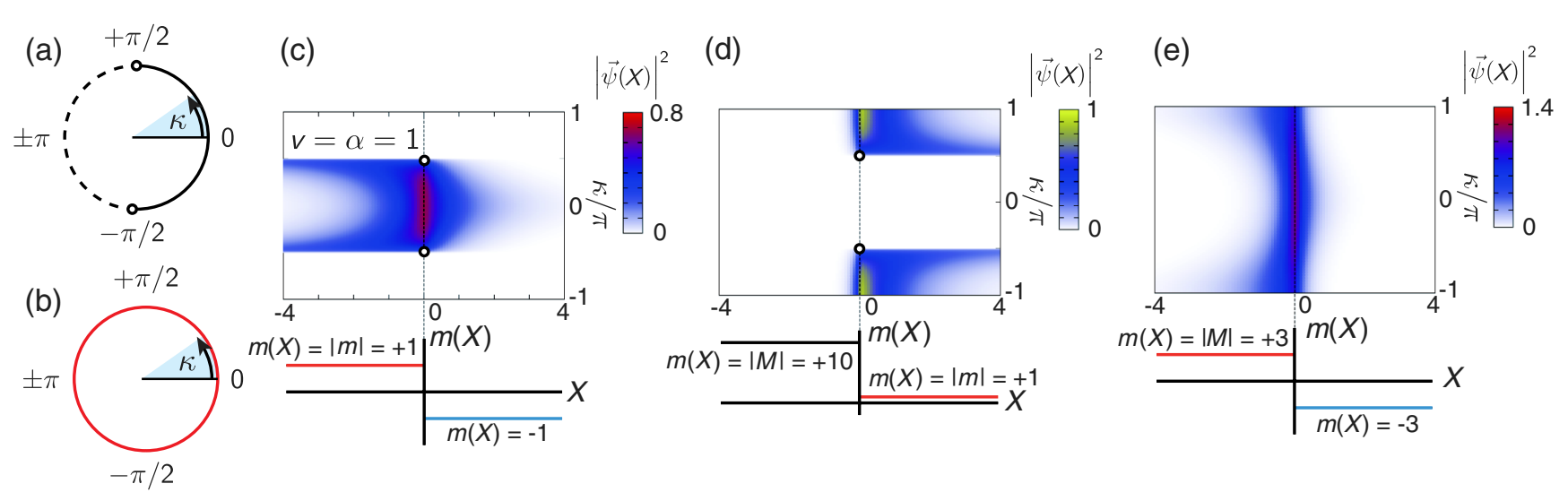

FIG. 5. Solutions for effective one-dimensional chiral Dirac equations (4). (a),(b) Simplified degrees of freedom $\kappa$ corresponding to one parameter representation of loci of zero modes on $\left(\kappa_{Y}, \kappa_{Z}\right)$. The solid (dashed) curve illustrates the loci of the domain-wall (surface) zero modes. White circles indicate the Weyl points. (a) The case before the pair annihilation of the Weyl points, corresponding to (c) and (d). On the other hand, (b) after the pair annihilation corresponds to (e), where the surface loci are eaten up by the domain-wall loci, and the domain-wall loci form a closed Fermi surface in the Brillouin zone. (c) Wave-function amplitude for a domain-wall state for the chiral Dirac equation (4) with $\alpha_{+}$in the plane of the real-space coordinate $X$ and momentum coordinate $\kappa$, with $v=\alpha_{+}=1$ and $m(x)=-\theta(+X)+\theta(-X)$. The "arc" state at the domain wall penetrates into one side of the bulk at the Weyl points. Another degenerate "arc" state localized in the other nearby side of the domain wall, obtained with $v=1$ and $\alpha_{-}=-1$, penetrates to the other side of the bulk (not shown). (d) Wave-function amplitude for a surface state for the chiral Dirac equation (4), with $v=\alpha=1$ and $m(x)=\theta(+X)+10 \theta(-X)$. The "arc" is formed in the missing part of the domain-wall arc. (e) Wave-function amplitude for a domainwall state for the chiral Dirac equation (4), with $v=\alpha=1$ and $m(x)=-3 \theta(+X)+3 \theta(-X)$. The domain-wall state now forms the closed loop of the Fermi surface without penetration into the bulk. For $|m(X)|>2$, there are no zero modes for surfaces.

a pair of chiral Dirac equations that describes 1D Chern insulators $[2,37]$ defined by

$$
\left\{\left[\alpha_{ \pm}(1-\cos \kappa)-m(X)\right] \hat{\sigma}_{z}+v i \hat{\sigma}_{y} \partial_{X}\right\} \vec{\psi}(\vec{X})=E \vec{\psi}(\vec{X}),
$$

where $\alpha_{ \pm}= \pm \alpha(\alpha>0)$ and $\kappa$ represents the degrees of freedom of $\kappa_{Y}$ and $\kappa_{Z}$. The two "Weyl" points appear at a $\kappa$ that satisfies $\alpha(1-\cos \kappa)-m(X)=0$ (see Fig. 5).

When we approach the pair annihilation of the Weyl points, the Fermi arcs are expected to shrink on the surface. On the contrary, as confirmed later numerically, the Fermi arcs on the domain walls become elongated by eating a part of the former arc on the surface. Furthermore, after the pair annihilation, they form a closed loop (an open Fermi line connected through equivalent Brillouin-zone boundaries). As is clear in the high-symmetry model [Eq. (14)], after the pair annihilation of the Weyl points, the surface between a vacuum and the domain is no longer a topological boundary: For a given $\kappa$, boundaries where the mass changes are classified in the terminology of the 1D Chern insulators. The topological invariant changes its sign at the domain walls as the 1D weak Chern insulators. (See also Sec. VIII for a detailed symmetry analysis.)

Here, we note that, although there exists a substantial similarity of the present Dirac Hamiltonian to the wellstudied Su-Schrieffer-Heeger Hamiltonian [39], namely, the chiral symmetry shared by both Hamiltonians, the latter effective Hamiltonian for polyacetylene and other conducting polymers additionally possesses the time-reversal and particle-hole symmetries. In addition to the difference in the symmetric properties, our 1D chiral Dirac equations describe the domain-wall states at a specified $\kappa$. By a variation of $\kappa$, they constitute $2 \mathrm{D}$ Fermi surfaces on the domain walls, while the edges of the Su-Schrieffer-Heeger model are genuinely zero-dimensional ones.

\section{UNRESTRICTED HARTREE-FOCK ANALYSIS}

The prediction based on the simple Dirac equations is confirmed by using fully unrestricted Hartree-Fock analysis (see Appendix F) of the original Hamiltonian (1) on the large supercell calculations with three different and typical domain walls, namely, $(01 \overline{1}),(100)$, and (111) domain walls, with a typical parameter set $U / t=4$ and $\zeta / t=-0.2$. (See Appendix $G$ for a definition of the supercells.) The self-consistent solution with optimized magnetic moment and charge distribution retains gapless domain-wall states, in general, and indeed on these three examples (see Fig. 6).

Moreover, these domain walls bring about uniform magnetizations perpendicular to themselves, which are defined as the sum of magnetic moments within the supercells. Surprisingly, the amplitude of these magnetizations per unit area of the domain wall does not depend on the direction of the domain wall and only depends on $m$ within numerical errors [Fig. 7(a)]. As a result, although the insertion of a single domain wall brings about a uniform nonzero magnetization, the total magnetization of a closed domain wall surrounding a domain may vanish. We note 
(a)

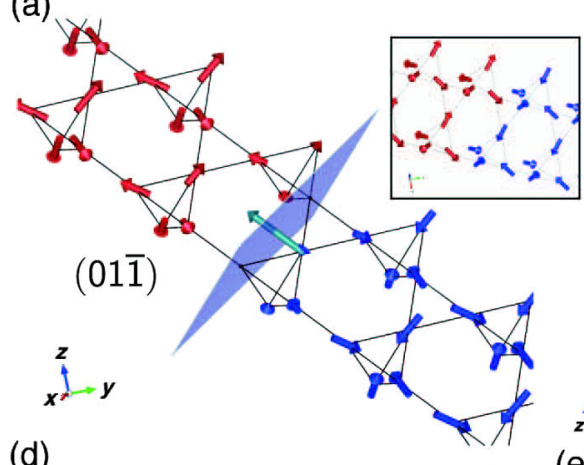

(d)

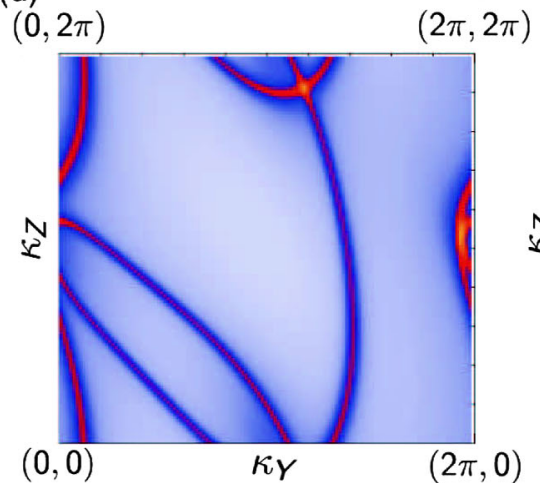

(b)

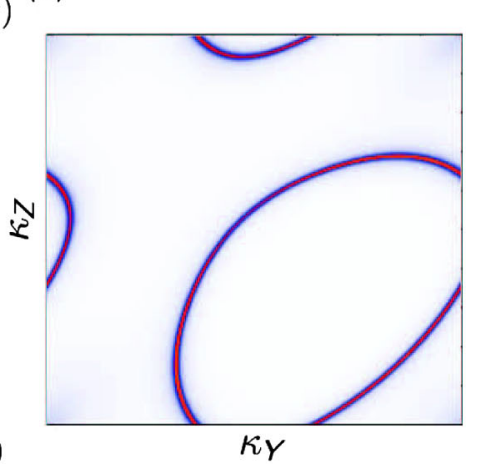

$(111)$ (c)
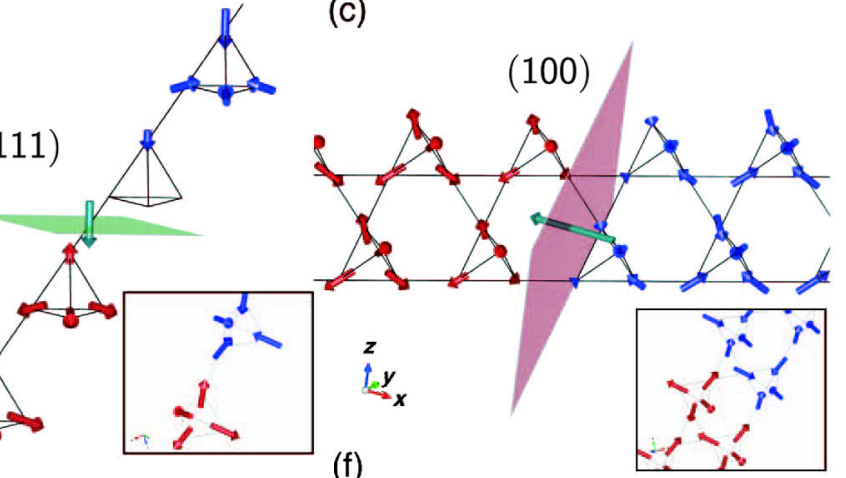

(f)

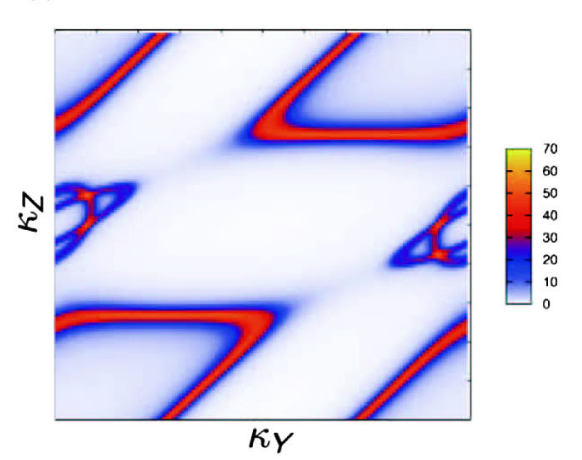

FIG. 6. Magnetic domain walls and domain-wall states. (a)-(c) Optimized magnetic structures for (011)), (111), and (100) domain walls, obtained from initial configurations shown in the insets, for $U / t=4, \zeta / t=-0.2$, and $k_{B} T / t=0.1$. The length and direction of the arrows are determined by optimized unrestricted Hartree-Fock solutions. We note that only a part of the supercells nearby the domain walls is illustrated. Uniform magnetization $m_{0}$ induced by the insertion of the domain walls and the intersection of the domain walls with the supercells are also illustrated as bold green arrows and shaded planes, respectively. (d)-(f) Spectral functions projected to the domain walls for (d) the (01̄) domain wall, (e) the (111) domain wall, and (f) the (100) domain wall, for $k_{B} T / t=0.1$, with a finite Lorentzian width $\delta=0.01 t$. At this temperature, bulk Weyl points do not exist any more. Every domain wall contributes to in-gap states at the chemical potential or the Fermi level, forming open two-dimensional Fermi surfaces. As a consequence of the pair annihilation of the Weyl points shown in Fig. 3(c), the Fermi arcs for the domain walls are now closed at this temperature. The complexity of the domainwall Fermi surfaces originates from the following fact: Depending on the orientation of the domain walls, there are many choices of how to connect the Weyl points with the loci of the domain-wall zero modes.

that the net magnetization of the whole system depends on termination of the system, as net magnetizations and/or electric polarizations of the usual antiferromagnets and/or ferroelectrics indeed depend on the termination of the systems. In this article, we only use the supercells tiled by tetrahedrons that are not sharing sites with each other (defined in Appendix C), for a clear-cut argument.

Here, we note that our tight-binding model is introduced as the simplest model for the holes in the $J_{\text {eff }}=1 / 2$ manifold of $R_{2} \mathrm{Ir}_{2} \mathrm{O}_{7}$. Therefore, we need to distinguish total angular momenta from magnetic moments of the physical spins. In this article, we use the $J_{\text {eff }}=1 / 2$ basis throughout and show total angular momenta as magnetic moments. The magnetic moments of the physical spins, therefore, align in the same direction with calculated total angular momenta and have an amplitude with $1 / 3$ of that of the total angular momenta.

The cancellation of the domain magnetizations is similar to that of a pair consisting of a spin soliton and an antisoliton sandwiching a domain of polyacetylene [39].
It becomes, however, incomplete, when external electric fields, lattice strain, defects, charged impurities, and/or doped carriers [as we see in Fig. 7(b)] exist. These incomplete cancellations are essentially the inverse effects of magnetostrain and/or magnetocharge responses [40].

\section{SYMMETRIC AND TOPOLOGICAL PROPERTIES}

The low-energy effective theory of the domain-wall states and the mean-field solutions for the domain walls in the tight-binding model have been discussed so far. Here, we show general properties of the domain-wall states that do not depend on the details of these theories: Symmetric properties and topological characterizations of these domain-wall states are given below. First, we show the symmetric properties both in the low-energy Dirac or Weyl equations and the mean-field solutions of the tight-binding model, which protect the degeneracy of the domain-wall states. Then, in an example of the (111) domain walls, we 

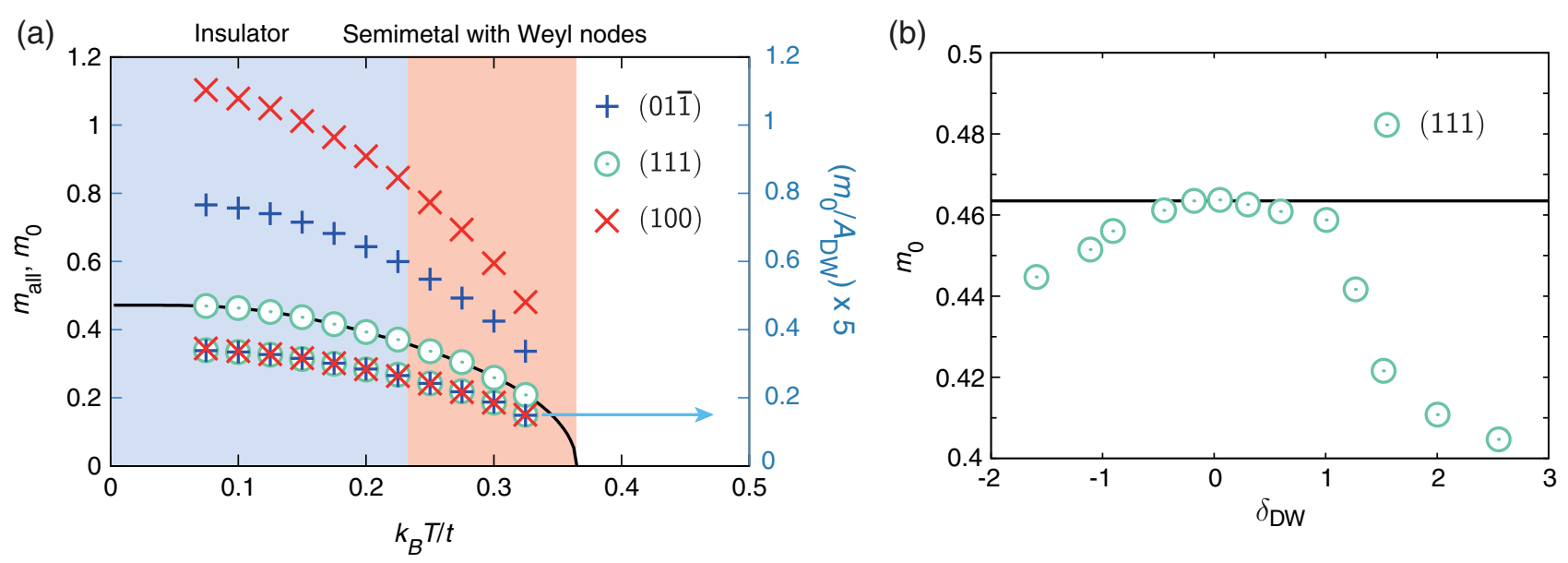

FIG. 7. Uniform magnetization $m_{0}$ induced around the domain walls. (a) Uniform magnetization $m_{0}$ induced around the domain walls per supercell shown as the top three symbols at each temperature for three different domain-wall planes, in comparison with the bulk allin- all-out magnetic ordered moment $m_{\text {all }}$ (solid black curve). The uniform magnetization per unit area $\left(a^{2}\right)$ of the domain walls $m_{0} / A_{\mathrm{DW}}$ is also shown as the three lowest symbols at each temperature. Here, the area of the intersection of a supercell with the domain walls $A_{\mathrm{DW}}$ for the $(01 \overline{1}),(111)$, and (100) domain walls are $8 \sqrt{2}, 4 \sqrt{3}$, and 16 in units of $a^{2}$, respectively. These three data points are almost on top of each other. (b) Doping dependence of the uniform magnetization of the (111) domain wall for $k_{B} T / t=0.1$. The doping $\delta_{\mathrm{DW}}$ is defined as an increase or decrease of the electron number per present supercell around the domain walls due to changes in the chemical potential. It shows that the uniform magnetization is insensitive to the charge doping around the domain wall, within $\left|\delta_{\mathrm{DW}}\right|<1$. When more than one electron is doped in the supercell around the domain wall, the reduction of the uniform magnetization becomes significant, with an asymmetry between electron and hole dopings.

explicitly demonstrate the existence of a weak topological number based on the translational and threefold-rotational symmetries of the (111) domain walls.

\section{A. Degeneracy protected by symmetries}

Both the low-energy effective description and the microscopic mean-field solution of the domain walls are invariant under certain symmetry operations. For the domain walls in the low-energy effective theories, the domain walls are trivially invariant under the inversion around the center of the domain walls $\hat{I}$ accompanied by the time-reversal operation $\hat{\Theta}$.

The domain walls in the microscopic mean-field solutions have more symmetric properties, which are detailed below. The $(01 \overline{1})$ domain walls in the mean-field solution are invariant under the inversion $\hat{I}$ around a site on the domain boundaries with the time-reversal operation $\hat{\Theta}$. The simultaneous twofold rotation of the lattice and the spins around the $(01 \overline{1})$ axis $\hat{C}_{2}^{(01 \overline{1})}$ with $\hat{\Theta}$ also leaves the $(01 \overline{1})$ domain walls invariant. The combination of a twofold rotation around the (001) axis $\hat{C}_{2}^{(001)}$ and $\hat{\Theta}$ leaves the (100) domain walls invariant. The twofold rotation around the (100) axis $\hat{C}_{2}^{(100)}$ also keeps the (100) domain walls unchanged. The (111) domain walls are invariant under the operations of the threefold rotation around the (111) axis $\hat{C}_{3}^{(111)}$ and the inversion with the time reversal $\hat{I} \hat{\Theta}$.

The invariance under the operation of the inversion with time reversal $\hat{I} \hat{\Theta}$ guarantees doubly degenerated Fermi surfaces at the (011) and (111) domain walls, while the invariance under the operation of $\hat{C}_{2}^{(001)} \hat{\Theta}$ guarantees the degeneracy for the (100) domain walls. In addition, the invariance under $\hat{C}_{2}^{(01 \overline{1})} \hat{\Theta}\left(\hat{C}_{2}^{(100)}\right)$ guarantees the degeneracy between the two $L$ points projected at the same momentum in the domain-wall Brillouin zones for the $(01 \overline{1})$ domain walls [the (100) domain walls].

\section{B. Hidden weak topological invariant}

In addition to the symmetric properties shown above, the topological nature of the (111) domain-wall states is characterized by the topological invariant of the zerodimensional class-A Chern insulators $[2,41,42]$. In other words, as detailed below, the domain-wall states are edge states of weak 1D Chern insulators embedded in the bulk.

First, we take an example of the $(1,1,1)$ domain walls. Here, we introduce the momentum frame $\left(k_{1}, k_{2}, k_{3}\right)$ through

$$
\begin{aligned}
\vec{k}^{T}= & \left(\frac{\pi}{4 a}, \frac{\pi}{4 a}, \frac{\pi}{4 a}\right)+k_{1}\left(\frac{1}{\sqrt{6}}, \frac{1}{\sqrt{6}},-\frac{2}{\sqrt{6}}\right) \\
& +k_{2}\left(-\frac{1}{\sqrt{2}}, \frac{1}{\sqrt{2}}, 0\right)+k_{3}\left(\frac{1}{\sqrt{3}}, \frac{1}{\sqrt{3}}, \frac{1}{\sqrt{3}}\right) .
\end{aligned}
$$

We set $\left(k_{1}, k_{2}\right)=(0,0)$ and drop the $k_{1}$ and $k_{2}$ dependence from $\hat{\mathcal{H}}_{0}(k)$ to concentrate on the projection of the $L$ point $(\pi / 4 a, \pi / 4 a, \pi / 4 a)$ on the $k_{1} k_{2}$ plane. The $k_{3}$ dependence is only noted as $\hat{\mathcal{H}}_{0}\left(k_{3}\right)$ for simplicity below. Then, we 
define the Hamiltonian that describes the sub-Hilbert space at the projection of the $L$ point or along the $\Gamma L$ line of the bulk Brillouin zone as

$\hat{H}_{0}^{(\Gamma L)}=\sum_{k_{3}} \overrightarrow{\hat{c}}_{k_{3}}^{\dagger} \hat{\mathcal{H}}_{0}\left(k_{3}\right) \overrightarrow{\hat{c}}_{k_{3}}=\sum_{x_{3}, x_{3}^{\prime}} \overrightarrow{\hat{c}}_{x_{3}}^{\dagger} \hat{\mathcal{H}}_{0}^{\prime}\left(x_{3}, x_{3}^{\prime}\right) \overrightarrow{\hat{c}}_{x_{3}^{\prime}}$,

where one-dimensional partial Fourier transformations are employed as $\overrightarrow{\hat{c}}_{x_{3}}^{\dagger}=L^{-1 / 2} \sum_{k_{3}} e^{i k_{3} x_{3}} \overrightarrow{\hat{c}}_{k_{3}}^{\dagger}$ for the number of the unit cell along the (111) direction, and the real-space 1D Hamiltonian matrix $\hat{\mathcal{H}}_{0}^{\prime}\left(x_{3}, x_{3}^{\prime}\right)$ is introduced. The 1D Hamiltonian $\hat{H}_{0}^{(\Gamma L)}$ embedded in the bulk Hamiltonian $\hat{H}_{0}$ describes a hidden $1 \mathrm{D}$ weak Chern insulator, as detailed below.

We prove that the one-dimensional Hamiltonian $\hat{H}_{0}^{(\Gamma L)}$ describes a hidden 1D weak topological insulator characterized by a zero-dimensional class-A topological invariant. The 1D weak topological insulator is protected by the translational symmetry along the (111) planes, the threefoldrotation symmetry $\left(C_{3}\right.$ rotation) around the (111) axis $\hat{C}_{3}^{(111)}$.

The translational symmetry prohibits scatterings among the eigenstates at the projection of the $L$ point $(\pi / 4 a, \pi / 4 a, \pi / 4 a)$ and eigenstates at other $k$ points in the $k_{1} k_{2}$ plane. Then, if a perturbation $\hat{\mathcal{V}}\left(x_{3}, x_{3}^{\prime}\right)$ keeps the threefold-rotation symmetry around the (111) axis and the mirror symmetry of the (111) plane, the deformed Hamiltonian

$$
\hat{H}^{(\Gamma L)}=\sum_{x_{3}, x_{3}^{\prime}} \overrightarrow{\hat{c}}_{x_{3}}^{\dagger}\left[\hat{\mathcal{H}}_{0}^{\prime}\left(x_{3}, x_{3}^{\prime}\right)+\hat{\mathcal{V}}\left(x_{3}, x_{3}^{\prime}\right)\right] \overrightarrow{\hat{c}}_{x_{3}^{\prime}}
$$

is characterized by a class-A topological invariant at $d=0$. Even after the introduction of the perturbation $\hat{\mathcal{V}}\left(x_{3}, x_{3}^{\prime}\right)$, the symmetric properties of the eigenstates under the $C_{3}$ rotation remain unchanged from those of the unperturbed Hamiltonian. Therefore, we classify the eigenstates of the perturbed Hamiltonian $\hat{H}^{(\Gamma L)}$ by the symmetric properties of the eigenstates of the unperturbed Hamiltonian $\hat{H}_{0}^{(\Gamma L)}$ as follows. The eigenstates of $\hat{\mathcal{H}}_{0}^{\prime}\left(x_{3}, x_{3}^{\prime}\right)$ are categorized into eight bands, which is evident in the spectrum of the Fouriertransformed Hamiltonian $\hat{\mathcal{H}}_{0}\left(k_{3}\right)$. By taking into account the fact that the $C_{3}$ rotation is a discrete symmetric operation, the symmetric property of each eigenstate is characterized by that of the eigenstates of $\hat{\mathcal{H}}_{0}\left(k_{3}=0\right)$. The eigenstates of $\hat{\mathcal{H}}_{0}\left(k_{3}=0\right)$ are classified into the four Zeemann-split doublets $2 E_{1 / 2 u} \oplus E_{1 / 2 g} \oplus E_{3 / 2 u}$.

There are $8 L$ eigenstates of the perturbed Hamiltonian $\hat{H}^{(\Gamma L)}$. Out of the $8 L$ eigenstates, $2 L$ eigenstates belong to $E_{3 / 2 u}$. Under the presence of the all-in-all-out orders, $E_{3 / 2 u}$ is split into two groups: $L$ states belonging to $E_{3 / 2 u}$ are located above the Fermi level, and the other $L$ states remain under the Fermi level in the bulk insulators. If the perturbation $\hat{\mathcal{V}}\left(x_{3}, x_{3}^{\prime}\right)$ keeps the $C_{3}$ rotation intact, there are no scatterings among the $2 L$ states labeled by the $E_{3 / 2 u}$ states and the other $6 L$ states, due to differences in the (111) component of angular momenta $m_{111}$, for these irreducible representations. The wave functions belonging to $2 E_{1 / 2 u} \oplus E_{1 / 2 g}$ are transformed as

$\hat{C}_{3}^{(111)}\left|\Phi ; m_{111}= \pm 1 / 2\right\rangle=e^{ \pm i \pi / 3}\left|\Phi ; m_{111}= \pm 1 / 2\right\rangle$,

while the wave functions belonging to $E_{3 / 2 u}$ are transformed under the operation of the $\hat{C}_{3}^{(111)}$ as

$$
\hat{C}_{3}^{(111)}\left|\Phi ; m_{111}= \pm 3 / 2\right\rangle=-\left|\Phi ; m_{111}= \pm 3 / 2\right\rangle .
$$

Here, we note the following fact: If the $L$ orbitals with $m_{111}=+1 / 2$ are occupied in the all-out phase, the $2 L$ orbitals with $m_{111}=-1 / 2$ are also occupied. When the time-reversal operation is applied, the $2 L$ orbitals with $m_{111}=+1 / 2$ and the $L$ orbitals with $m_{111}=-1 / 2$ are necessarily occupied. In addition, the $C_{3}$-rotational symmetry prohibits the scatterings among orbitals with different (111) components of the angular momentum $m_{111}$.

To describe the structure of the spectrum at the projected $L$ point in detail, matrices are defined as

$$
\left(H_{0}^{(\Gamma L)}\right)_{i, j}=\left\langle 0\left|\hat{c}_{x \nu \sigma} \hat{H}_{0}^{(\Gamma L)} \hat{c}_{y \mu \tau}\right| 0\right\rangle
$$

and

$$
\left(H^{(\Gamma L)}\right)_{i, j}=\left\langle 0\left|\hat{c}_{x \nu \sigma} \hat{H}^{(\Gamma L)} \hat{c}_{y \mu \tau}\right| 0\right\rangle,
$$

where $i=(x, \nu, \sigma)$ and $j=(y, \mu, \tau)$. Then, the matrix representation of the unperturbed Hamiltonian is diagonalized as

$$
H_{0}^{(\Gamma L)}=U_{8 L \times 8 L}\left[\begin{array}{ccc}
\boldsymbol{D}_{2 L} & \mathbf{0}_{2 L \times 3 L} & \mathbf{0}_{2 L \times 3 L} \\
\mathbf{0}_{3 L \times 2 L} & \boldsymbol{D}_{3 L}^{(+)} & \mathbf{0}_{3 L \times 3 L} \\
\mathbf{0}_{3 L \times 2 L} & \mathbf{0}_{3 L \times 3 L} & \boldsymbol{D}_{3 L}^{(-)}
\end{array}\right] U_{8 L \times 8 L}^{\dagger},
$$

where $\boldsymbol{D}_{2 L}$ and $\boldsymbol{D}_{3 L}^{( \pm)}$are $2 L \times 2 L$ and $3 L \times 3 L$ diagonal matrices, respectively. The submatrices $\boldsymbol{D}_{3 L}^{( \pm)}$represent the eigenvalues of the eigenstates that are labeled by the $2 E_{1 / 2 u} \oplus E_{1 / 2 g}$ states. The sub-Hilbert space of the orbitals with $m_{111}=+1 / 2\left(m_{111}=-1 / 2\right)$ are represented by the submatrix $\boldsymbol{D}_{3 L}^{(+)}\left(\boldsymbol{D}_{3 L}^{(-)}\right)$. The $C_{3}$-rotational symmetry that prohibits the scattering among the three sub-Hilbert spaces represented by $\boldsymbol{D}_{2 L}$ and $\boldsymbol{D}_{3 L}^{( \pm)}$leads to an important consequence: The unitary matrix $U_{8 L \times 8 L}$ transforms the perturbed Hamiltonian matrix $H^{(\Gamma L)}$ into the blockdiagonalized form as

$$
H^{(\Gamma L)}=U_{8 L \times 8 L}\left[\begin{array}{ccc}
\boldsymbol{M}_{2 L} & \mathbf{0}_{2 L \times 3 L} & \mathbf{0}_{2 L \times 3 L} \\
\mathbf{0}_{3 L \times 2 L} & \boldsymbol{M}_{3 L}^{(+)} & \mathbf{0}_{3 L \times 3 L} \\
\mathbf{0}_{3 L \times 2 L} & \mathbf{0}_{3 L \times 3 L} & \boldsymbol{M}_{3 L}^{(-)}
\end{array}\right] U_{8 L \times 8 L}^{\dagger} .
$$


Here, we call the number of the occupied orbitals that belong to $\boldsymbol{M}_{3 L}^{( \pm)}$in the all-out phase $m_{3 L}^{( \pm)}$, respectively. Then, if the system remains gapped, the set of the numbers of the occupied orbitals $\left(m_{3 L}^{(+)}, m_{3 L}^{(-)}\right)$is invariant under any perturbation that keeps the $C_{3}$-rotational symmetry around the (111) axis.

Therefore, in case of the magnetic domain walls that keep the $C_{3}$-rotational symmetry around the (111) axis intact, the numbers of the occupied orbitals $\left(m_{3 L}^{(+)}, m_{3 L}^{(-)}\right)$ give a zero-dimensional topological invariant. If the filling of the system is kept at half-filling, the conservation of the electrons leads to $m_{3 L}^{(+)}+m_{3 L}^{(-)}=3 L$. Therefore, one of them gives us a $\boldsymbol{Z}$ topological invariant classified in the zero-dimensional class A [41,42]. Here, we note that the all-in-all-out ordered phases of the pyrochlore iridium oxides do not possess the chiral, particle-hole, and timereversal symmetries by themselves.

The robust domain-wall states must exist when the weak topological invariant changes at the domain walls. The changes in the invariant indeed occur. If we remind readers that the eigenstates classified by $E_{1 / 2 g}$ are split into two groups under the presence of the all-in- all-out orders, the zero-dimensional topological invariant $m_{3 L}^{(+)}$necessarily changes from $2 L$ to $L$, or $L$ to $2 L$, across the domain walls.

The interchanges in the occupation of $m_{111}=+1 / 2$ states and $m_{111}=-1 / 2$ states correspond to the switching of the location of the zeroth Landau levels from the all-out domains to the all-in domains. Furthermore, as detailed in Appendix E, the eigenstates belonging to $E_{1 / 2 g}$ with $m_{111}=-1 / 2$ indeed participate in the wave function of the zeroth Landau level.

\section{Gapless excitations at domain-wall states}

The 1D weak Chern insulators embedded in the bulk allin-all-out ordered phases guarantee the existence of the ingap states. The robust in-gap states, however, do not necessarily lead to gapless quasiparticle excitations at the domain walls. Below, we show that the metallic domain-wall states are guaranteed by the degeneracy of the domain-wall states protected by the symmetries of the domain walls introduced in Sec. VIII A.

The appearance of the protected in-gap states due to interchange of the eigenstates above and below the Fermi level imposes a constraint on the number of eigenstates belonging to the conduction, valence, and in-gap states: At the projected $L$ point, there are $4 L-\ell$ conduction and valence states, while there are $2 \ell$ in-gap states, where $\ell$ is some integer. The low-energy effective 1D Dirac and 3D Weyl equations give us the precise value of $\ell$, which is invariant due to the topological protection under any perturbations that keep the symmetry of the domain walls. For the (111) domain walls, the number of the in-gap states is given as $2 \ell=2$.

The additional symmetry of the domain walls, namely, the invariance under $\hat{I} \hat{\Theta}$ for the (111) domain walls, protects the twofold degeneracy of the in-gap states. Even away from the projected $L$ point, the in-gap-state degeneracy is kept, as illustrated in Fig. 8(b). In addition, the in-gap states never disappear unless they are merged into the bulk Bloch states.

Then, if we assume that there are no gapless excitations, the conduction and valence bands contain $4 L^{3}+L^{2}$ and $4 L^{3}-L^{2}$ eigenstates, respectively, or $4 L^{3}-L^{2}$ and $4 L^{3}+L^{2}$ eigenstates, respectively, for the system with the $L^{3}$ unit cells. Thus, if there are no gapless excitations at the domain walls, the domain walls require $L^{2}$ electron or hole dopings that inevitably induce macroscopic electric polarizations. It also prohibits us from keeping the system at half-filling. Therefore, the domain-wall states inevitably offer gapless quasiparticle excitations at half-filling and/or without macroscopic electric polarizations.

In contrast, if the symmetry that protects the degeneracy of the domain-wall states is broken, the fully gapped states at half-filling are realized by opening gaps at the domainwall states, as schematically illustrated in Fig. 8(c). The degeneracy lifting and gap opening may indeed occur at the surface between the bulk and vacuum because at the surface, the required symmetry $[\hat{I} \hat{\Theta}$ for the (111) domain wall] is broken.

The domain-wall states are not chiral in total, although each domain-wall band may be characterized by its clockwise or counterclockwise chiral spin texture. These chiral textures cancel each other. As a result, the domain-wall Fermi surfaces seem to be trivially paramagnetic ones.

\section{24 Weyl nodes}

Here, we show that, even if the 24 Weyl nodes appear as shown in $\mathrm{LSDA}+\mathrm{SO}+U$ [6], the weak topological invariant is unchanged. As already mentioned in Sec. III, the 24 Weyl nodes can be induced by certain perturbations that keep the bulk lattice symmetry and, at least, the invariance of the system under $\hat{C}_{3}^{(111)}$. For example, the level scheme at the $L$ point is controlled by introducing third-neighbor hoppings between two sites connected by the real-space vectors with amplitudes $2 \sqrt{2} a$, such as $(2 a, 0,2 a)$ and its transformations under the symmetric operations belonging to the tetrahedron point group $T_{d}$.

Such perturbations shift the relative energy of $2 E_{1 / 2 u}$, $E_{1 / 2 g}$, and $E_{3 / 2 u}$ at the $L$ point, while the perturbations introduce a constant energy shift at the $\Gamma$ point, independently of the orbital classification. Indeed, the $E_{1 / 2 g}$ state with $m_{111}=+1 / 2$ below the Fermi level and the $E_{1 / 2 u}$ state with $m_{111}=+1 / 2$ above the Fermi level can be forced to touch each other at the $L$ point in the all-out phases $(m>0)$. As detailed in Ref. [6], the level cross between these two states induces the 24 Weyl nodes. When the magnetic ordered moment grows further, the 24 Weyl nodes become gapped, as also shown in Ref. [6]. Here, we note that, although the third-neighbor hoppings induce the 


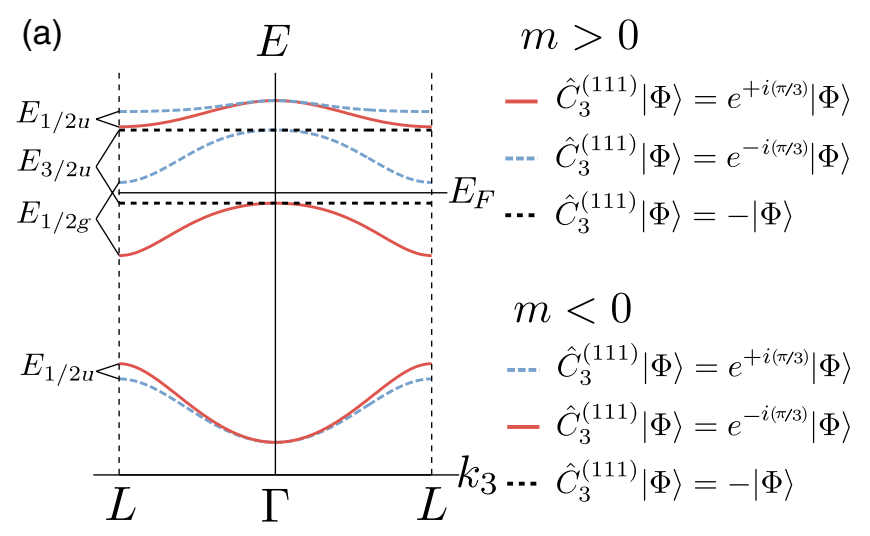

(b)

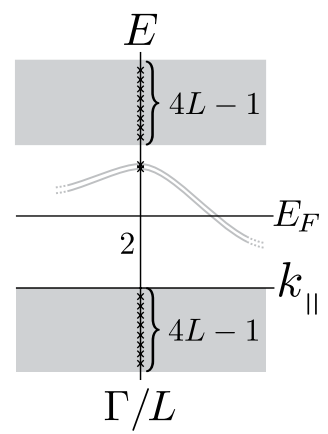

(c)

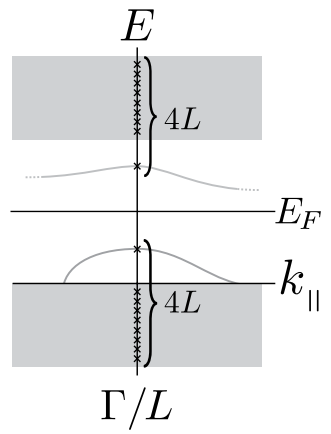

FIG. 8. (a) Classification of the eigenstates at the projected $L$ point. For illustrative purposes, the eigenstates are shown under the translational invariance along the (111) axis or the $\Gamma L$ line. These eigenstates are invariant under the threefold rotation $\hat{C}_{3}^{(111)}$ and, thus, labeled by the eigenvalues of the operator $\hat{C}_{3}^{(111)}$. Here, the eigenstates are $e^{ \pm i(\pi / 3)}$ and -1 . For the all-out domain $(m>0)$, the solid red (dotted blue) curves represent the eigenstates with the eigenvalue $e^{+i(\pi / 3)}\left(e^{-i(\pi / 3)}\right)$. After applying the time-reversal operation, namely, in the all-in domain $(m<0)$, these eigenstates are switched as the eigenvalue $e^{-i(\pi / 3)}\left(e^{+i(\pi / 3)}\right)$ corresponds to the solid red (dashed blue) curves. (b) Schematic energy spectrum of a bulk with a single domain wall along momentum $k_{\|}$that is perpendicular to $k_{3}$, with the protected twofold degeneracy of the domain-wall states. These doubly degenerated domain-wall dispersions can be characterized by clockwise and counterclockwise chiral spin textures. The domain-wall states by themselves do not possess the inversion symmetry around the projected $\Gamma$ and/or $L$ points. (c) Schematic possible energy spectrum along $k_{\|}$without the degeneracy of the domain-wall states.

24 Weyl nodes, further-neighbor hoppings are required for the realization of Weyl semimetals.

Even when the Weyl semimetals with the 24 Weyl nodes are realized, an important fact is led: After the gap opening of the 24 Weyl nodes, the number of the unoccupied orbitals with $m_{111}=+1 / 2$ is unchanged in comparison with the original energy spectrum after the pair annihilation of the eight Weyl nodes [depicted in Fig. 8(a)]. Therefore, the 24 Weyl nodes do not leave any trace after they are gapped out, in contrast to the eight Weyl nodes that leave the edge states of the 1D weak Chern insulators after their pair annihilations. Irrespective of the existence or the absence of the 24 Weyl points, after the magnetic ordered moment grows, the topological invariants defined by such as the number of $E_{1 / 2 u}$ states with $m_{111}=+1 / 2$ are determined from the physics of the eight Weyl points clarified here and are preserved, even when the eight Weyl points do not show up near the Fermi level.

\section{DISCUSSION AND COMPARISON WITH EXPERIMENTS}

Here, we discuss implications and comparisons of the present theory and experimental results observed in $R_{2} \mathrm{Ir}_{2} \mathrm{O}_{7}$. We find consistencies between our domain-wall theory and the experimental indications: By cooling under magnetic fields, magnetic domain walls are formed and pinned at their favorable impurity or disorder sites to optimize the net magnetization along the external magnetic fields and thus generate a nonzero magnetization with the difference between zero- and nonzero-field coolings in the experiments [10,13]. From Fig. 7(b), we find that $n_{\mathrm{ex}}$ excess carriers per unit cell induce uniform magnetization $m_{0}$ created by insertion of the magnetic domain walls roughly up to approximately $g \mu_{B} n_{\text {ex }}$, when the domain-wall concentration is $n_{\text {ex }}$ (namely, the averaged domain size is approximately $n_{\mathrm{ex}}^{-1}$ unit cells). A realistic value $n_{\mathrm{ex}} \sim 10^{-3}$ explains the peculiar uniform magnetization (approximately $10^{-3} \mu_{B}$ /unit cell) universally observed experimentally [10]. Self-doping may also spontaneously stabilize such a stable domain structure. The smaller magnetization for polycrystals [13] is consistent because magnetic domains are wiped out more easily than those in single crystals. Here, we note that, in contrast to the concentration of magnetic domain walls, the concentration of impurities or disorders does not necessarily depend on whether the sample is a polycrystal or a single crystal. The larger hysteresis for stoichiometric samples [13] is simply ascribed to stronger all-in-all-out order.

The conduction on the domain wall becomes dominating at low temperatures after the elimination of the bulk Weyl electrons: Strong sample dependence [13] and hysteresis in the magnetization sweep [14] at the lowest temperatures support this view. Our gapless electronic states are doubly degenerate and localized at the opposite sides of the domain wall from each other, which generate mutual scatterings and cause weak but notable Anderson localization. We note that the double degeneracy is the consequence of the gapless solutions obtained from $\hat{h}^{(+)}$and $\hat{h}^{(-)}$. It is an intriguing future problem how the degeneracy is lifted. Because of the degeneracy, the domain-wall Fermi surface seems to be a paramagnetic one. However, once its degeneracy is lifted by external magnetic fields, two Zeeman-split Fermi surfaces can be chiral, for example, for (111) domain walls. Each split Fermi surface has momentum-dependent spin polarization, as expected in noncoplanar itinerant magnets, and will show geometric 
anomalous Hall conductivities. A tempting explanation of the large negative magnetoresistance for $\mathrm{Nd}$ or $\mathrm{Gd}$ compounds $[14,19]$ is the fluctuating ferromagnetic moment of $\mathrm{Nd}$ induced by $m_{0}$ at zero field, which scatters carriers at domain walls similarly to the double-exchange mechanism. It is desired to further understand them more quantitatively for a better magnetic control of the transport.

We here further discuss the novelty of the present domain-wall excitation. We note that the analogy of the present domain wall with the solitons in polyacetylene is helpful as an intuitive concept but should be understood with caution: It is impossible to straightforwardly generalize solitons in polyacetylene to 3D systems and to create 2D metallic states with these solitons because the basic equations are not the same. Furthermore, the topological classification of the polyacetylene, which is actually labeled as BDI in the Élie Cartan's classification scheme [2], is different from the present ones classified as 1D weak Chern insulators because of the crucial differences in the symmetry and spatial dimensionality, which generate different classes of topological phase. As far as we know, magnetic domain walls have never been clarified in the light of the one-dimensional (weak) Chern insulators, possibly embedded in the seemingly trivial insulators. The possibility of such an insulator has never been pointed out in theoretical and experimental studies on magnetism of not only the pyrochlore iridium oxides but also the magnetic domains, in general. From the viewpoint of the physics of the magnetic domain and domain wall in the long history, it has never been anticipated that it can have a topological character with metallic conduction distinct from the bulk. Therefore, the present domain wall is a new type of magnetic excitation.

In addition to the purely scientific advances, our result shows that the domain-wall states in pyrochlore iridium oxides open a new way of building conducting 2D electron systems that are controllable through magnetic fields. Control of electric transports by external magnetic and/ or electric fields has been a central idea of electronics and spintronics. The domain-wall Fermi surfaces predicted in our present work can be not only swept by external fields, after fabrication of samples, but also offer possible anomalous Hall metals with reduced backscatterings (in other words, high mobility), due to the background noncollinear all-in- all-out magnetic orders. Possible interplay between the domain-wall states and magnetic moments of magnetic ions may already be observed in $\mathrm{Gd}$ or $\mathrm{Nd}$ pyrochlore iridium oxides as a huge negative magnetoresistance.

\section{ACKNOWLEDGMENTS}

We thank Taka-hisa Arima for fruitful discussions. Y. Y. thanks Moyuru Kurita for an enlightening discussion in the early stage of the present study. Y. Y. also thanks Takahiro Misawa for his comments on the topological classification of the 1D Dirac equations and Satoru Hayami for drawing his attention to Ref. [36]. This work is financially supported by MEXT HPCI Strategic Programs for Innovative Research (SPIRE) (hp130007) and the Computational Materials Science Initiative (CMSI). Numerical calculation was partly carried out at the Supercomputer Center, Institute for Solid State Physics, University of Tokyo. This work was also supported by Grants-in-Aid for Scientific Research (No. 22104010, No. 22340090, and No. 23740261) from MEXT, Japan.

\section{APPENDIX A: DERIVATION OF LOW-ENERGY HAMILTONIAN}

We study Weyl electrons by using the Hamiltonian (1), which describes hole states of the $J_{\text {eff }}=1 / 2$ manifold of iridium atoms. After the mean-field decoupling given in Appendix F, and using the unrestricted Hartree-Fock solution of Eq. (1) given by the all-in-all-out magnetic order, we replace the $U$ term with the mean-field one with the order parameter $m$.

We begin with a Fourier-transformed form of the Hartree-Fock Hamiltonian given by an $8 \times 8$ Hamiltonian. By extracting a low-energy Hilbert space around the Fermi level, it is reduced to a $6 \times 6$ Hamiltonian. If we use $k$-group terminology at the $\Gamma$ point, we extract a $T_{2 g} \otimes E_{1 / 2}$ manifold of a double group $T_{d}$ from $\left(T_{2 g} \oplus A_{1 g}\right) \otimes E_{1 / 2}$.

Next, we further extract a $4 \times 4$ low-energy part of the $6 \times 6$ effective Hamiltonian. This corresponds to extraction of the $G_{3 / 2}$ manifold (or $J_{3 / 2}$ manifold) from $T_{2 g} \otimes E_{1 / 2}=E_{5 / 2} \oplus G_{3 / 2}$, in the $k$-group terminology.

\section{1. $8 \times 8$ Hamiltonian}

The Fourier-transformed form of the $8 \times 8$ Hamiltonian (1) after the Hartree-Fock approximation is given by

$$
\hat{H}_{0}=\sum_{\vec{k}} \sum_{\nu=1, \ldots, 4} \sum_{\alpha, \beta=\uparrow, \downarrow} \hat{c}_{\vec{k} \nu \alpha}^{\dagger} \hat{\mathcal{H}}_{0}(\vec{k}) \hat{c}_{\vec{k} \mu \beta},
$$

and $\hat{\mathcal{H}}_{0}=\hat{\mathcal{K}}_{0}+\hat{\mathcal{Z}}_{0}+\hat{\mathcal{M}}_{0}$, with $\hat{\mathcal{K}}_{0}$ being the kinetic term proportional to $t$ as

$$
\hat{\mathcal{K}}_{0}(\vec{k})=-2 t \hat{\sigma}_{0}\left[\begin{array}{cccc}
0 & \cos \left(k_{x}-k_{y}\right) & \cos \left(k_{y}-k_{z}\right) & \cos \left(k_{z}+k_{x}\right) \\
\cos \left(k_{x}-k_{y}\right) & 0 & \cos \left(k_{z}-k_{x}\right) & \cos \left(k_{y}+k_{z}\right) \\
\cos \left(k_{y}-k_{z}\right) & \cos \left(k_{z}-k_{x}\right) & 0 & \cos \left(k_{x}+k_{y}\right) \\
\cos \left(k_{z}+k_{x}\right) & \cos \left(k_{y}+k_{z}\right) & \cos \left(k_{x}+k_{y}\right) & 0
\end{array}\right]
$$


$\hat{\mathcal{Z}}_{0}$ being the spin-orbit term proportional to $\zeta$ as

$$
\hat{\mathcal{Z}}_{0}(\vec{k})=2 i \zeta\left[\begin{array}{cccc}
0 & +\frac{\hat{\sigma}_{x}+\hat{\sigma}_{y}}{\sqrt{2}} \cos \left(k_{x}-k_{y}\right) & -\frac{\hat{\sigma}_{y}+\hat{\sigma}_{z}}{\sqrt{2}} \cos \left(k_{y}-k_{z}\right) & +\frac{\hat{\sigma}_{z}-\hat{\sigma}_{x}}{\sqrt{2}} \cos \left(k_{z}+k_{x}\right) \\
-\frac{\hat{\sigma}_{x}+\hat{\sigma}_{y}}{\sqrt{2}} \cos \left(k_{x}-k_{y}\right) & 0 & +\frac{\hat{\sigma}_{z}+\hat{\sigma}_{x}}{\sqrt{2}} \cos \left(k_{z}-k_{x}\right) & +\frac{\hat{\sigma}_{y}-\hat{\sigma}_{z}}{\sqrt{2}} \cos \left(k_{y}+k_{z}\right) \\
+\frac{\hat{\sigma}_{y}+\hat{\sigma}_{z}}{\sqrt{2}} \cos \left(k_{y}-k_{z}\right) & -\frac{\hat{\sigma}_{z}+\hat{\sigma}_{x}}{\sqrt{2}} \cos \left(k_{z}-k_{x}\right) & 0 & +\frac{\hat{\sigma}_{x}-\hat{\sigma}_{y}}{\sqrt{2}} \cos \left(k_{x}+k_{y}\right) \\
-\frac{\hat{\sigma}_{z}-\hat{\sigma}_{x}}{\sqrt{2}} \cos \left(k_{x}+k_{z}\right) & -\frac{\hat{\sigma}_{y}-\hat{\sigma}_{z}}{\sqrt{2}} \cos \left(k_{y}+k_{z}\right) & -\frac{\hat{\sigma}_{x}-\hat{\sigma}_{y}}{\sqrt{2}} \cos \left(k_{x}+k_{y}\right) & 0
\end{array}\right],
$$

and $\hat{\mathcal{M}}_{0}$ being the Hartree-Fock term of the all-in-all-out magnetic order proportional to $m$

$$
\hat{\mathcal{M}}_{0}=\frac{m}{\sqrt{3}}\left[\begin{array}{cccc}
+\hat{\sigma}_{x}-\hat{\sigma}_{y}+\hat{\sigma}_{z} & 0 & 0 & 0 \\
0 & -\hat{\sigma}_{x}+\hat{\sigma}_{y}+\hat{\sigma}_{z} & 0 & 0 \\
0 & 0 & +\hat{\sigma}_{x}+\hat{\sigma}_{y}-\hat{\sigma}_{z} & 0 \\
0 & 0 & 0 & -\hat{\sigma}_{x}-\hat{\sigma}_{y}-\hat{\sigma}_{z}
\end{array}\right] \text {. }
$$

\section{Reduction from $8 \times 8$ to $6 \times 6$ Hamiltonian}

By assuming $|\zeta| / t, m / t, k^{2} \ll 1$, the leading-order terms of the $6 \times 6$ low-energy effective Hamiltonian $\hat{\mathcal{H}}_{1}=$ $\hat{\mathcal{K}}_{1}+\hat{\mathcal{Z}}_{1}+\hat{\mathcal{M}}_{1}$ are extracted by using a projection

$$
\hat{\mathcal{P}}_{4 \times 3}=\frac{1}{2}\left[\begin{array}{ccc}
+1 & -1 & +1 \\
-1 & +1 & +1 \\
+1 & +1 & -1 \\
-1 & -1 & -1
\end{array}\right]
$$

with

$$
\begin{aligned}
\hat{\mathcal{K}}_{1}=\hat{\mathcal{P}}_{4 \times 3}^{T} \hat{\mathcal{K}}_{0} \hat{\mathcal{P}}_{4 \times 3}= & -2 t \hat{\sigma}_{0}\left(\cos k_{x} \cos k_{y}\left[\begin{array}{ccc}
-1 & 0 & 0 \\
0 & -1 & 0 \\
0 & 0 & +1
\end{array}\right]+\cos k_{y} \cos k_{z}\left[\begin{array}{ccc}
+1 & 0 & 0 \\
0 & -1 & 0 \\
0 & 0 & -1
\end{array}\right]+\cos k_{z} \cos k_{x}\left[\begin{array}{ccc}
-1 & 0 & 0 \\
0 & +1 & 0 \\
0 & 0 & -1
\end{array}\right]\right) \\
& \left.-2 t \hat{\sigma}_{0}\left[\begin{array}{ccc}
0 & \sin k_{x} \sin k_{y} & \sin k_{z} \sin x_{x} \\
\sin k_{x} \sin k_{y} & 0 & \sin k_{y} \sin k_{z} \\
\sin k_{z} \sin k_{x} & \sin k_{y} \sin k_{z} & 0
\end{array}\right]\right) \\
= & -2 t \hat{\sigma}_{0}\left[\begin{array}{ccc}
-1+k_{x}^{2} & k_{x} k_{y} & k_{x} k_{z} \\
k_{y} k_{x} & -1+k_{y}^{2} & k_{y} k_{z} \\
k_{z} k_{x} & k_{z} k_{y} & -1+k_{z}^{2}
\end{array}\right]+\mathcal{O}\left(k^{3}\right)=2 t \mathbf{1}_{6}-2 t\left(\vec{k} \hat{\sigma}_{0}\right) \otimes\left(\vec{k} \hat{\sigma}_{0}\right)+\mathcal{O}\left(k^{3}\right)
\end{aligned}
$$

and

$$
\hat{\mathcal{Z}}_{1}=\hat{\mathcal{P}}_{4 \times 3}^{T} \hat{\mathcal{Z}}_{0} \hat{\mathcal{P}}_{4 \times 3}=2 \sqrt{2} i \zeta\left[\begin{array}{ccc}
0 & -\hat{\sigma}_{z} & +\hat{\sigma}_{y} \\
+\hat{\sigma}_{z} & 0 & -\hat{\sigma}_{x} \\
-\hat{\sigma}_{y} & +\hat{\sigma}_{x} & 0
\end{array}\right]+\mathcal{O}\left(\zeta k^{2}\right)
$$


The all-in-all-out mean field $\hat{\mathcal{M}}_{0}$ is projected to the lowenergy subspace as

$$
\hat{\mathcal{M}}_{1}=\hat{\mathcal{P}}_{4 \times 3}^{T} \hat{\mathcal{M}}_{0} \hat{\mathcal{P}}_{4 \times 3}=\frac{m}{\sqrt{3}}\left[\begin{array}{ccc}
0 & \hat{\sigma}_{z} & \hat{\sigma}_{y} \\
\hat{\sigma}_{z} & 0 & \hat{\sigma}_{x} \\
\hat{\sigma}_{y} & \hat{\sigma}_{x} & 0
\end{array}\right] .
$$

\section{a. Reduction from $6 \times 6$ to $4 \times 4$}

Then, we extract a $4 \times 4$ Hamiltonian from $\hat{\mathcal{H}}_{1}$ by using a unitary transformation consisting of irreducible representations $E_{5 / 2}$ and $G_{3 / 2}$ of the double group $T_{d}$

$$
\hat{\mathcal{U}}_{J}=\left[\begin{array}{ccc}
-\frac{1}{\sqrt{3}} \hat{\sigma}_{x} & -\frac{1}{\sqrt{2}} \hat{\sigma}_{z} & +\frac{i}{\sqrt{6}} \hat{\sigma}_{y} \\
-\frac{1}{\sqrt{3}} \hat{\sigma}_{y} & -\frac{i}{\sqrt{2}} \hat{\sigma}_{0} & -\frac{i}{\sqrt{6}} \hat{\sigma}_{x} \\
-\frac{1}{\sqrt{3}} \hat{\sigma}_{z} & 0 & +\frac{2}{\sqrt{6}} \hat{\sigma}_{0}
\end{array}\right] .
$$

$$
\hat{\kappa}(\vec{k})=\left[\begin{array}{c}
\frac{k_{x}^{2}+k_{y}^{2}}{2} \hat{\sigma}_{0} \\
-\frac{k_{x}^{2}-k_{y}^{2}}{2 \sqrt{3}} \hat{\sigma}_{x}-\frac{k_{x} k_{y}}{\sqrt{3}} \hat{\sigma}_{y}-\frac{k_{z} k_{x}}{\sqrt{3}} \hat{\sigma}_{z}-i \frac{k_{y} k_{z}}{\sqrt{3}} \hat{\sigma}_{0}
\end{array}\right.
$$

The effective spin-orbit coupling and the all-in-all-out mean field are transformed as

$$
\tilde{\mathcal{Z}}_{2}=\hat{\mathcal{U}}_{J}^{\dagger} \hat{\mathcal{Z}}_{1} \hat{\mathcal{U}}_{J}=2 \sqrt{2} \zeta\left[\begin{array}{ccc}
-2 \hat{\sigma}_{0} & 0 & 0 \\
0 & \hat{\sigma}_{0} & 0 \\
0 & 0 & \hat{\sigma}_{0}
\end{array}\right]
$$

and

$$
\tilde{\mathcal{M}}_{2}=\hat{\mathcal{U}}_{J}^{\dagger} \hat{\mathcal{M}}_{1} \hat{\mathcal{U}}_{J}=\left[\begin{array}{ccc}
0 & 0 & 0 \\
0 & 0 & +i m \hat{\sigma}_{x} \\
0 & -i m \hat{\sigma}_{x} & 0
\end{array}\right]
$$

For $\zeta<0$, by counting the number of states, it becomes clear that the chemical potential is located within the $G_{3 / 2}$ manifold to keep the electron density at half-filling, in other words, one electron per site. Here, the reduction to the $4 \times 4$ Hamiltonian $\hat{\mathcal{H}}_{2} \equiv \hat{\mathcal{K}}_{2}+\hat{\mathcal{Z}}_{2}+\hat{\mathcal{M}}_{2}$ is achieved by ignoring the off-diagonal term $\overrightarrow{\hat{\nu}}^{\dagger}(\vec{k})$, which generates negligible corrections of $\mathcal{O}\left(t k^{4} / 6 \sqrt{2}|\zeta|\right)$. Here, $\hat{\mathcal{K}}_{2}=\hat{\kappa}(\vec{k})$, while $\hat{\mathcal{Z}}_{2}$ and $\hat{\mathcal{M}}_{2}$ are the lower right $4 \times 4$ components of $\tilde{\mathcal{Z}}_{2}$ and $\tilde{\mathcal{M}}_{2}$, respectively. We take the notation for the tight-binding part as $\hat{h}_{4 \times 4} \equiv \hat{\mathcal{K}}_{2}+\hat{\mathcal{Z}}_{2}$, where $\hat{h}_{4 \times 4}$ can be rewritten in a compact form as
The first and second columns of $\hat{\mathcal{U}}_{J}$ correspond to the $E_{5 / 2}$ irreducible representation, and the other columns correspond to the $G_{3 / 2}$ irreducible representation. Then, the kinetic term $\mathcal{K}_{1}$ is transformed as follows:

$$
\begin{aligned}
\hat{\mathcal{U}_{J}^{\dagger}} \hat{\mathcal{K}}_{1} \hat{\mathcal{U}}_{J} & =2 t \mathbf{1}_{6}-2 t \hat{\mathcal{U}} \dagger\left(\vec{k} \hat{\sigma}_{0}\right) \otimes\left(\vec{k} \hat{\sigma}_{0}\right) \hat{\mathcal{U}}_{J} \\
& =2 t \mathbf{1}_{6}-2 t\left[\begin{array}{cc}
\frac{1}{3} k^{2} \hat{\sigma}_{0} & \overrightarrow{\hat{\nu}} \dagger(\vec{k}) \\
\overrightarrow{\hat{\nu}}(\vec{k}) & \hat{\kappa}(\vec{k})
\end{array}\right],
\end{aligned}
$$

where

$$
\begin{aligned}
\overrightarrow{\hat{\nu}}^{\dagger}(\vec{k})= & \left(\frac{k_{z} k_{x}}{\sqrt{6}} \hat{\sigma}_{0}+i \frac{k_{y} k_{z}}{\sqrt{6}} \hat{\sigma}_{z}-i \frac{k_{x}^{2}-k_{y}^{2}}{\sqrt{6}} \hat{\sigma}_{y}\right. \\
& \left.+i \frac{2 k_{x} k_{y}}{\sqrt{6}}, \frac{k^{2}-3 k_{z}^{2}}{3 \sqrt{2}} \hat{\sigma}_{z}-\frac{k_{z} k_{x}}{\sqrt{2}} \hat{\sigma}_{x}-\frac{k_{y} k_{z}}{\sqrt{2}} \hat{\sigma}_{y}\right)
\end{aligned}
$$

and

$$
\left.\begin{array}{c}
-\frac{k_{x}^{2}-k_{y}^{2}}{2 \sqrt{3}} \hat{\sigma}_{x}-\frac{k_{x} k_{y}}{\sqrt{3}} \hat{\sigma}_{y}-\frac{k_{z} k_{x}}{\sqrt{3}} \hat{\sigma}_{z}+i \frac{k_{y} k_{z}}{\sqrt{3}} \hat{\sigma}_{0} \\
\frac{k^{2}+3 k_{z}^{2}}{6} \hat{\sigma}_{0}
\end{array}\right] .
$$

where

$\vec{d}(\vec{k})^{T}=-\left(\frac{k_{y} k_{z}}{\sqrt{3}}, \frac{k_{z} k_{x}}{\sqrt{3}}, \frac{k_{x} k_{y}}{\sqrt{3}}, \frac{k_{x}^{2}-k_{y}^{2}}{2 \sqrt{3}}, \frac{3 k_{z}^{2}-k^{2}}{6}\right)$

and a vector of Dirac matrices $\vec{\Gamma}^{T}=\left(\hat{\Gamma}^{1}, \hat{\Gamma}^{2}, \hat{\Gamma}^{3}, \hat{\Gamma}^{4}, \hat{\Gamma}^{5}\right)$, which give time-reversal-symmetric terms for the Hilbert space of the $4 \times 4$ Hamiltonian. The above Hamiltonian is nothing but a variation of the Luttinger Hamiltonian. Dirac matrices used here are defined as follows:

$$
\begin{aligned}
& \hat{\Gamma}^{1}=\left[\begin{array}{cc}
0 & -i \hat{\sigma}_{0} \\
+i \hat{\sigma}_{0} & 0
\end{array}\right], \\
& \hat{\Gamma}^{2}=\left[\begin{array}{cc}
0 & +\hat{\sigma}_{z} \\
+\hat{\sigma}_{z} & 0
\end{array}\right], \\
& \hat{\Gamma}^{3}=\left[\begin{array}{cc}
0 & +\hat{\sigma}_{y} \\
+\hat{\sigma}_{y} & 0
\end{array}\right], \\
& \hat{\Gamma}^{4}=\left[\begin{array}{cc}
0 & +\hat{\sigma}_{x} \\
+\hat{\sigma}_{x} & 0
\end{array}\right],
\end{aligned}
$$




$$
\hat{\Gamma}^{5}=\left[\begin{array}{cc}
+\hat{\sigma}_{0} & 0 \\
0 & -\hat{\sigma}_{0}
\end{array}\right]
$$

where $\hat{\sigma}_{0}$ is the two-dimensional identity matrix and $\hat{\sigma}_{a}(a=x, y, z)$ are the Pauli matrices. From these five Dirac matrices, the other Dirac matrices representing timereversal symmetry-breaking perturbations are defined as

$$
\hat{\Gamma}^{a b}=\left[\hat{\Gamma}^{a}, \hat{\Gamma}^{b}\right] / 2 i,
$$

where, for example, the all-in- all-out magnetic order parameter is represented by $\hat{\mathcal{M}}_{2}=m \hat{\Gamma}^{54}$.

\section{APPENDIX B: GREEN'S FUNCTION}

By calculating the Green's functions discussed below, electronic spectra for the $4 \times 4$ Hamiltonian become accessible. The Green's function $\hat{G}_{4 \times 4}$ for the Bloch Hamiltonian $\hat{h}_{4 \times 4}$ is defined by

$$
\begin{aligned}
\hat{G}_{4 \times 4}(\vec{k}, \omega)^{-1} & =(\omega+\mu) \hat{\sigma}_{0} \otimes \hat{\tau}_{0}-\hat{h}_{4 \times 4}(\vec{k})-m \hat{\Gamma}^{54} \\
& =\varpi(\vec{k}, \omega) 1_{4}+2 t \vec{d}(\vec{k}) \cdot \overrightarrow{\hat{\Gamma}}-m \hat{\Gamma}^{54}
\end{aligned}
$$

where $\varpi(\vec{k}, \omega)=\omega+\mu-2 t+2 \sqrt{2}|\zeta|+2 t k^{2} / 3$.

Inverting the right-hand side of Eq. (B1), we obtain the Green's function as follows:

$$
\begin{aligned}
\hat{G}_{4 \times 4}= & {\left[\varpi 1_{4}-2 t \vec{d} \cdot \overrightarrow{\hat{\Gamma}}+m \hat{\Gamma}^{54}\right] \frac{\left[\varpi^{2}-4 t^{2}|\vec{d}|^{2}-m^{2}\right] \mathbf{1}_{4}-4 m t\left[d_{3} \hat{\Gamma}^{21}+d_{2} \hat{\Gamma}^{13}+d_{1} \hat{\Gamma}^{32}\right]}{\left[\varpi^{2}-4 t^{2}|\vec{d}|^{2}-m^{2}\right]^{2}-16 m^{2} t^{2}\left[d_{1}^{2}+d_{2}^{2}+d_{3}^{2}\right]} } \\
= & {\left[\frac{\frac{\mathbf{1}_{4}}{2}+\frac{-2 t \vec{d} \cdot \overrightarrow{\hat{\Gamma}}+m \hat{\Gamma}^{54}}{2 E_{+}}}{\varpi-E_{+}}+\frac{\frac{\mathbf{1}_{4}}{2}-\frac{-2 \vec{d} \cdot \overrightarrow{\hat{\Gamma}}+m \hat{\Gamma}^{54}}{2 E_{+}}}{\varpi+E_{+}}\right]\left[\frac{\mathbf{1}_{4}}{2}-\operatorname{sgn}(m) \frac{d_{3} \hat{\Gamma}^{21}+d_{2} \hat{\Gamma}^{13}+d_{1} \hat{\Gamma}^{32}}{2 \sqrt{d_{1}^{2}+d_{2}^{2}+d_{3}^{2}}}\right] } \\
& +\left[\frac{\frac{\mathbf{1}_{4}}{2}+\frac{-2 \vec{d} \cdot \overrightarrow{\hat{\Gamma}}+m \hat{\Gamma}^{54}}{2 E_{-}}}{\varpi-E_{-}}+\frac{\frac{\mathbf{1}_{4}}{2}-\frac{-2 t \vec{d} \cdot \vec{\Gamma}+m \hat{\Gamma}^{54}}{2 E_{-}}}{\varpi+E_{-}}\right]\left[\frac{\mathbf{1}_{4}}{2}+\operatorname{sgn}(m) \frac{d_{3} \hat{\Gamma}^{21}+d_{2} \hat{\Gamma}^{13}+d_{1} \hat{\Gamma}^{32}}{2 \sqrt{d_{1}^{2}+d_{2}^{2}+d_{3}^{2}}}\right] .
\end{aligned}
$$

Here, we omit the $\vec{k}$ and $\omega$ dependences above and define the functions $E_{ \pm}$as

$$
E_{ \pm}(\vec{k})=\sqrt{4 t^{2}|\vec{d}(\vec{k})|^{2}+m^{2} \pm 4|m| t \sqrt{d_{1}(\vec{k})^{2}+d_{2}(\vec{k})^{2}+d_{3}(\vec{k})^{2}}} .
$$

For the above calculation of the Green's function, the following two identities are useful:

$$
|\vec{d}(\vec{k})|=|\vec{k}|^{2} / 3
$$

and

$$
[\vec{d}(\vec{k}) \cdot \overrightarrow{\hat{\Gamma}}]^{2}=|\vec{d}(\vec{k})|^{2}
$$

\section{APPENDIX C: DOMAIN WALLS}

For each of three domain walls, namely, $(01 \overline{1}),(111)$, and (100) domain walls, we introduce coordinate transformations given as follows.

For the $(01 \overline{1})$ domain wall, we introduce a new oblique coordinate $(X, Y, Z)$, with $(Y, Z)$ parallel to the domainwall plane and corresponding momentum frame $\left(\kappa_{X}, \kappa_{Y}, \kappa_{Z}\right)$

$$
\vec{r}=X\left[\begin{array}{c}
0 \\
+2 a \\
-2 a
\end{array}\right]+Y\left[\begin{array}{c}
0 \\
-2 a \\
-2 a
\end{array}\right]+Z\left[\begin{array}{c}
-4 a \\
+2 a \\
+2 a
\end{array}\right]
$$

and

$$
\vec{k}=\kappa_{X}\left[\begin{array}{c}
0 \\
+1 / 4 a \\
-1 / 4 a
\end{array}\right]+\kappa_{Y}\left[\begin{array}{c}
-1 / 4 a \\
-1 / 4 a \\
-1 / 4 a
\end{array}\right]+\kappa_{Z}\left[\begin{array}{c}
-1 / 4 a \\
0 \\
0
\end{array}\right] .
$$

For the (111) domain wall,

$$
\vec{r}=X\left[\begin{array}{c}
-2 a \\
0 \\
-2 a
\end{array}\right]+Y\left[\begin{array}{c}
-2 a \\
+2 a \\
0
\end{array}\right]+Z\left[\begin{array}{c}
0 \\
+2 a \\
-2 a
\end{array}\right]
$$


$\vec{k}=\kappa_{X}\left[\begin{array}{l}-1 / 4 a \\ -1 / 4 a \\ -1 / 4 a\end{array}\right]+\kappa_{Y}\left[\begin{array}{l}-1 / 4 a \\ +1 / 4 a \\ +1 / 4 a\end{array}\right]+\kappa_{Z}\left[\begin{array}{c}+1 / 4 a \\ +1 / 4 a \\ -1 / 4 a\end{array}\right]$.

For the (100) domain wall,

$$
\vec{r}=X\left[\begin{array}{c}
+2 a \\
+2 a \\
0
\end{array}\right]+Y\left[\begin{array}{c}
0 \\
+4 a \\
+4 a
\end{array}\right]+Z\left[\begin{array}{c}
0 \\
0 \\
+4 a
\end{array}\right]
$$

and

$\vec{k}=\kappa_{X}\left[\begin{array}{c}+1 / 2 a \\ 0 \\ 0\end{array}\right]+\kappa_{Y}\left[\begin{array}{c}-1 / 4 a \\ +1 / 4 a \\ 0\end{array}\right]+\kappa_{Z}\left[\begin{array}{c}+1 / 4 a \\ -1 / 4 a \\ +1 / 4 a\end{array}\right]$.

\section{APPENDIX D: 1D DIRAC EQUATIONS}

In this section, we derive 1D Dirac equations that describe low-energy single-electron states of the $4 \times 4$ effective Hamiltonian derived above. By using the derived 1D Dirac equations, we obtain an analytic description of domain-wall states. For illustrative purposes, we focus on

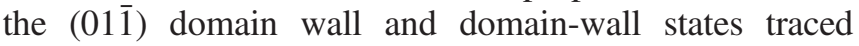

back to the bulk Weyl electrons around $\vec{k}_{\mathrm{Weyl}}=$ $\pm \sqrt{|m| / 2 t}(1,1,1)^{T}$ and $\vec{k}_{\text {Weyl }}= \pm \sqrt{|m| / 2 t}(-1,1,1)^{T}$.

Along a symmetry axis parallel to $\vec{k}=(+1,+1,+1)$, the $4 \times 4$ Hamiltonian is diagonalized by the following unitary matrix

$$
\hat{\mathcal{U}}_{(1,1,1)}=\left[\begin{array}{llll}
-a i & -b i & +b i & +a i \\
-b \vartheta & +a \vartheta & -a \vartheta & +b \vartheta \\
+b \vartheta^{*} & -a \vartheta^{*} & -a \vartheta^{*} & +b \vartheta^{*} \\
+a & +b & +b & +a
\end{array}\right]
$$

where

$$
\begin{gathered}
\vartheta=\frac{1+i}{\sqrt{2}}, \\
a=\frac{\sqrt{\sqrt{3}+1}}{2 \cdot 3^{1 / 4}}, \\
b=\frac{\sqrt{\sqrt{3}-1}}{2 \cdot 3^{1 / 4}} .
\end{gathered}
$$

It is useful to list up the unitary transformation of the matrices $\hat{\Gamma}^{i}(i=1,2,3,4,5)$ and $m \hat{\Gamma}^{54}$ :

$$
\begin{aligned}
& \hat{\mathcal{U}}_{(1,1,1)}^{\dagger} \hat{\Gamma}^{1} \hat{\mathcal{U}}_{(1,1,1)}=\left[\begin{array}{cccc}
+2 a b\left(\vartheta+\vartheta^{*}\right) & -2 a^{2} \vartheta^{*}+2 b^{2} \vartheta & 0 & 0 \\
-2 a^{2} \vartheta+2 b^{2} \vartheta^{*} & -2 a b\left(\vartheta+\vartheta^{*}\right) & 0 & 0 \\
0 & 0 & +2 a b\left(\vartheta+\vartheta^{*}\right) & +2 a^{2} \vartheta-2 b^{2} \vartheta^{*} \\
0 & 0 & +2 a^{2} \vartheta^{*}-2 b^{2} \vartheta & -2 a b\left(\vartheta+\vartheta^{*}\right)
\end{array}\right] \\
& =\left[\begin{array}{cccc}
+1 / \sqrt{3} & -1 / \sqrt{6}+i / \sqrt{2} & 0 & 0 \\
-1 / \sqrt{6}-i / \sqrt{2} & -1 / \sqrt{3} & 0 & 0 \\
0 & 0 & +1 / \sqrt{3} & +1 / \sqrt{6}+i / \sqrt{2} \\
0 & 0 & +1 / \sqrt{6}-i / \sqrt{2} & -1 / \sqrt{3}
\end{array}\right], \\
& \hat{\mathcal{U}}_{(1,1,1)}^{\dagger} \hat{\Gamma}^{2} \hat{\mathcal{U}}_{(1,1,1)}=\left[\begin{array}{cccc}
+2 a b\left(\vartheta+\vartheta^{*}\right) & -2 a^{2} \vartheta+2 b^{2} \vartheta^{*} & 0 & 0 \\
-2 a^{2} \vartheta^{*}+2 b^{2} \vartheta & -2 a b\left(\vartheta+\vartheta^{*}\right) & 0 & 0 \\
0 & 0 & +2 a b\left(\vartheta+\vartheta^{*}\right) & +2 a^{2} \vartheta^{*}-2 b^{2} \vartheta \\
0 & 0 & +2 a^{2} \vartheta-2 b^{2} \vartheta^{*} & -2 a b\left(\vartheta+\vartheta^{*}\right)
\end{array}\right] \\
& =\left[\begin{array}{cccc}
+1 / \sqrt{3} & -1 / \sqrt{6}-i / \sqrt{2} & 0 & 0 \\
-1 / \sqrt{6}+i / \sqrt{2} & -1 / \sqrt{3} & 0 & 0 \\
0 & 0 & +1 / \sqrt{3} & +1 / \sqrt{6}-i / \sqrt{2} \\
0 & 0 & +1 / \sqrt{6}+i / \sqrt{2} & -1 / \sqrt{3}
\end{array}\right],
\end{aligned}
$$




$$
\begin{gathered}
\hat{\mathcal{U}}_{(1,1,1)}^{\dagger} \hat{\Gamma}^{3} \hat{\mathcal{U}}_{(1,1,1)}=\left[\begin{array}{cccc}
+2\left(a^{2}-b^{2}\right) & +4 a b & 0 & 0 \\
+4 a b & -2\left(a^{2}-b^{2}\right) & 0 & 0 \\
0 & 0 & +2\left(a^{2}-b^{2}\right) & -4 a b \\
0 & 0 & -4 a b & -2\left(a^{2}-b^{2}\right)
\end{array}\right] \\
=\left[\begin{array}{ccccc}
+1 / \sqrt{3} & +2 / \sqrt{6} & 0 & 0 \\
+2 / \sqrt{6} & -1 / \sqrt{3} & 0 & 0 \\
0 & 0 & +1 / \sqrt{3} & -2 / \sqrt{6} \\
0 & 0 & -2 / \sqrt{6} & -1 / \sqrt{3}
\end{array}\right], \\
\hat{\mathcal{U}}_{(1,1,1)}^{\dagger} \hat{\Gamma}^{4} \hat{\mathcal{U}}_{(1,1,1)}=\left[\begin{array}{cccc}
0 & 0 & 0 & +i \\
0 & 0 & +i & 0 \\
0 & -i & 0 & 0 \\
-i & 0 & 0 & 0
\end{array}\right], \quad \text { (D11) } \\
\text { Then, the vector } \vec{d}(\vec{k})=\left(d_{1}, d_{2}, d_{3}, d_{4}, d_{5}\right)^{T} \text { is trat } \vec{G}_{X}+\frac{\kappa_{Y}}{\pi} \vec{G}_{Y}+\frac{\kappa_{Z}}{\pi} \vec{G}_{Z}
\end{gathered}
$$

Then, the vector $\vec{d}(\vec{k})=\left(d_{1}, d_{2}, d_{3}, d_{4}, d_{5}\right)^{T}$ is transformed as

$$
\begin{gathered}
d_{1}=\frac{-1}{\sqrt{3}}\left(-\kappa_{X}^{2}+\kappa_{Y}^{2}\right), \\
d_{2}=\frac{-1}{\sqrt{3}}\left\{+\left(\kappa_{Y}+\kappa_{Z}\right) \kappa_{X}+\left(\kappa_{Y}+\kappa_{Z}\right) \kappa_{Y}\right\}, \\
d_{3}=\frac{-1}{\sqrt{3}}\left\{-\left(\kappa_{Y}+\kappa_{Z}\right) \kappa_{X}+\left(\kappa_{Y}+\kappa_{Z}\right) \kappa_{Y}\right\}, \\
d_{4}=\frac{-1}{2 \sqrt{3}}\left(-\kappa_{X}^{2}+2 \kappa_{Y} \kappa_{X}+\kappa_{Z}^{2}+2 \kappa_{Y} \kappa_{Z}\right), \\
d_{5}=\frac{-1}{6}\left(\kappa_{X}^{2}+6 \kappa_{Y} \kappa_{X}-\kappa_{Z}^{2}-2 \kappa_{Y} \kappa_{Z}\right) .
\end{gathered}
$$

Since $k$-independent and diagonal terms are absorbed

First, we consider the case of $\vec{k}_{\mathrm{Weyl}}=$ $\pm \sqrt{|m| / 2 t}(1,1,1)^{T}$. For the $(01 \overline{1})$ domain wall, we introduce a new momentum frame $\vec{\kappa}=\left(\kappa_{X}, \kappa_{Y}, \kappa_{Z}\right)^{T}$ as into the chemical potential renormalization, $\hat{\mathcal{H}}_{2}=$ $\hat{h}_{4 \times 4}(\vec{k})+\hat{\mathcal{M}}_{2}$ may be rewritten after the unitary transformation $\hat{\mathcal{U}}_{(1,1,1)}$ as

$$
\begin{aligned}
\hat{\mathcal{U}}_{(1,1,1)}^{\dagger} \hat{h}_{4 \times 4} \hat{\mathcal{U}}_{(1,1,1)}= & -\frac{2}{3} t k^{2} \hat{\sigma}_{0} \otimes \hat{\tau}_{0}-2 t \hat{\mathcal{U}}_{(1,1,1)}^{\dagger} \vec{d} \cdot \overrightarrow{\hat{\Gamma}} \hat{\mathcal{U}}_{(1,1,1)} \\
= & -\frac{2}{3} t k^{2} \hat{\sigma}_{0} \otimes \hat{\tau}_{0}-2 t\left\{-\kappa_{Y}^{2} \hat{\sigma}_{z} \otimes \hat{\tau}_{0}+\kappa_{Y} \kappa_{X}\left[\hat{\sigma}_{x} \otimes \hat{\tau}_{x}+\frac{1}{\sqrt{3}} \hat{\sigma}_{x} \otimes \hat{\tau}_{y}\right]\right. \\
& +\frac{\kappa_{Y}+\kappa_{Z}}{\sqrt{3}} \kappa_{X}\left[\sqrt{\frac{3}{2}} \hat{\sigma}_{x} \otimes \hat{\tau}_{z}-\frac{1}{\sqrt{2}} \hat{\sigma}_{y} \otimes \hat{\tau}_{0}\right] \\
& +\kappa_{Z}\left(\kappa_{Z}+2 \kappa_{Y}\right)\left[-\frac{1}{6} \hat{\sigma}_{x} \otimes \hat{\tau}_{x}+\frac{1}{2 \sqrt{3}} \hat{\sigma}_{x} \otimes \hat{\tau}_{y}\right] \\
& \left.-\frac{1}{\sqrt{3}} \kappa_{Z} \kappa_{Y}\left[\frac{2}{\sqrt{3}} \hat{\sigma}_{z} \otimes \hat{\tau}_{0}+\frac{1}{\sqrt{6}} \hat{\sigma}_{x} \otimes \hat{\tau}_{z}+\frac{1}{\sqrt{2}} \hat{\sigma}_{y} \otimes \hat{\tau}_{0}\right]\right\}
\end{aligned}
$$


and

$$
\hat{\mathcal{U}}_{(1,1,1)}^{\dagger} \hat{\mathcal{M}}_{2} \hat{\mathcal{U}}_{(1,1,1)}=m \hat{\sigma}_{0} \otimes \hat{\tau}_{z}
$$

where it is indeed diagonal at $\vec{k}_{\text {Weyl }}= \pm \sqrt{|m| / 2 t}(1,1,1)^{T}$, which translates to $\kappa_{X}=\kappa_{Z}=0$ and $\kappa_{Y}=$ $\kappa_{0} / \sqrt{3} \equiv \pm \sqrt{|m| / 2 t}$.
If $m>0$, the second and third components constitute the Weyl electrons. In other words, for $m>0$, the diagonal matrix $+2 t \kappa_{Y}^{2} \hat{\sigma}_{z} \otimes \hat{\tau}_{0}+m \hat{\sigma}_{0} \otimes \hat{\tau}_{z}$ is nonzero for the first and fourth components and possibly has zero eigenvalues at $\kappa_{X}=\kappa_{Z}=0$ only for the second and third components, namely, at $2 t \kappa_{Y}^{2}=m$. Then, by extracting the second and third components, the $2 \times 2$ Hamiltonian is obtained as

$$
\begin{aligned}
\hat{h}_{\Gamma \vec{k}_{\text {weyl }}}^{(+)}\left(\kappa_{X}, \delta \kappa_{Y}, \kappa_{Z}\right)= & -\frac{2 t}{3} k^{2}+\left[\begin{array}{llll}
0 & 0 & 0 & 0 \\
0 & 1 & 0 & 0 \\
0 & 0 & 1 & 0 \\
0 & 0 & 0 & 0
\end{array}\right] \hat{\mathcal{U}}_{(1,1,1)}^{\dagger}\left(-2 t \vec{d} \cdot \overrightarrow{\hat{\Gamma}}+m \hat{\Gamma}^{54}\right) \hat{\mathcal{U}}_{(1,1,1)}\left[\begin{array}{cccc}
0 & 0 & 0 & 0 \\
0 & 1 & 0 & 0 \\
0 & 0 & 1 & 0 \\
0 & 0 & 0 & 0
\end{array}\right] \\
= & -2 t \kappa_{Y}^{2}-\frac{4 t}{3} \kappa_{Z} \kappa_{Y}-\frac{2 t}{3} \kappa_{Z}^{2}-2 t\left(\kappa_{Y}^{2}+\frac{2}{3} \kappa_{Z} \kappa_{Y}\right) \hat{\sigma}_{z}-\frac{4 t}{\sqrt{3}} \kappa_{Y} \kappa_{X} \hat{\sigma}_{y}+\frac{2 t}{3} \kappa_{Z}\left(\kappa_{Z}+2 \kappa_{Y}\right) \hat{\sigma}_{x}+m \hat{\sigma}_{z} \\
\simeq & -2 t\left(\frac{\kappa_{0}}{\sqrt{3}}\right)^{2}-4 t \frac{\kappa_{0}}{\sqrt{3}} \delta \kappa_{Y}-\frac{4 t}{3 \sqrt{3}} \kappa_{0} \kappa_{Z}-2 t\left[\left(\frac{\kappa_{0}}{\sqrt{3}}\right)^{2}+2 \frac{\kappa_{0}}{\sqrt{3}} \delta \kappa_{Y}+\frac{2}{3 \sqrt{3}} \kappa_{0} \kappa_{Z}\right] \hat{\sigma}_{z}-\frac{4 t}{3} \kappa_{0} \kappa_{X} \hat{\sigma}_{y} \\
& +\frac{4 t}{3 \sqrt{3}} \kappa_{0} \kappa_{Z} \hat{\sigma}_{x}+m \hat{\sigma}_{z} .
\end{aligned}
$$

If $m<0$, zero eigenvalues may appear only for the first and fourth components. By extracting the first and fourth components, the $2 \times 2$ Hamiltonian has a form similar to Eq. (D22) as

$$
\hat{h}_{\Gamma \vec{k}_{\mathrm{Wey}}}^{(-)}\left(\kappa_{X}, \delta \kappa_{Y}, \kappa_{Z}\right)=4 t \frac{\kappa_{0}}{\sqrt{3}}\left\{-\left(\delta \kappa_{Y}+\frac{1}{3} \kappa_{Z}\right) \hat{\sigma}_{0}+\left(\delta \kappa_{Y}+\frac{1}{3} \kappa_{Z}\right) \hat{\sigma}_{z}-\frac{1}{\sqrt{3}} \hat{\sigma}_{y} \kappa_{X}+\frac{1}{3} \kappa_{Z} \hat{\sigma}_{x}+\frac{m(X)+|m|}{4 t \kappa_{0} / \sqrt{3}} \hat{\sigma}_{z}\right\} .
$$

Then, surface and domain-wall states are obtained by solving the Dirac Hamiltonians (D22) and (D23). For simplicity, we concentrate on a pair of the Weyl points $\vec{k}_{\text {Weyl }}= \pm(\sqrt{|m| / 2 t}, \sqrt{|m| / 2 t}, \sqrt{|m| / 2 t})^{T}$ and on a surface or domain perpendicular to $(0,+1,-1)$, namely, a $(01 \overline{1})$ surface or domain. In the following discussion, we take the coordination axis along $(0,+1,-1)$ as the $X$ axis. Around these two Weyl points, low-energy quasiparticle excitations are described by the lowest-order $\vec{k} \cdot \vec{p}$-type Hamiltonian $\hat{h}_{\Gamma \vec{k}_{\mathrm{Wey}}}^{(+)}$up to the linear order in $-i \partial_{X}, \delta \kappa_{Y}$, and $\kappa_{Z}$

$\hat{h}_{\Gamma \vec{k}_{\text {Weyl }}^{(+)}}\left(-i \partial_{X}, \delta \kappa_{Y}, \kappa_{Z}\right)=4 t \frac{\kappa_{0}}{\sqrt{3}}\left\{-\left(\delta \kappa_{Y}+\frac{\kappa_{Z}}{3}\right) \hat{\sigma}_{0}+\frac{\kappa_{Z}}{3} \hat{\sigma}_{x}+\frac{i}{\sqrt{3}} \hat{\sigma}_{y} \partial_{X}+\left[-\left(\delta \kappa_{Y}+\frac{\kappa_{Z}}{3}+\frac{|m|}{4 t \kappa_{0} / \sqrt{3}}\right)+\frac{m(X)}{4 t \kappa_{0} / \sqrt{3}}\right] \hat{\sigma}_{z}\right\}$,

for $m(X)=+|m|$, where we introduce a new momentum frame and replace $\kappa_{X}$ with $-i \partial_{X}$. Here, the pair of Weyl points is given as $\vec{k}_{\text {Weyl }}=-\kappa_{0}(1 / \sqrt{3}, 1 / \sqrt{3}, 1 / \sqrt{3})^{T}$, with $\kappa_{0}= \pm \sqrt{3|m| / 2 t}$.

Then, the two-component one-dimensional Dirac equation

$$
\hat{h}_{\Gamma \vec{k}_{\mathrm{Weyl}}}^{(+)}\left(-i \partial_{X}, \delta \kappa_{Y}, \kappa_{Z}\right)\left[\begin{array}{l}
\psi_{1}(X) \\
\psi_{2}(X)
\end{array}\right]=E\left[\begin{array}{l}
\psi_{1}(X) \\
\psi_{2}(X)
\end{array}\right]
$$

gives a description of the bound states on the surface or domain walls by carefully choosing the $X$-dependent "mass" term $m(X)$ as follows. Here, we note that the all-out (all-in) domain is described by $m(X)=+|m|$ $[m(X)=-|m|]$. We also remind the readers that, for a large enough order parameter $|m|$, the Weyl points are annihilated in pairs and the bulk system becomes a trivial magnetic insulator with a charge excitation gap. Note that the mass term

$$
m(X)=\left\{\begin{array}{cc}
+|m| & (X<0) \\
-|m| & (0<X)
\end{array}\right.
$$

gives a magnetic domain wall at $X=0$, while the mass term

$$
m(X)= \begin{cases}+|M| & (X<0) \\ +|m| & (0<X)\end{cases}
$$

with $|M| \gg|m|$ mimicking a surface between a vacuum $(X<0)$ and the bulk $(X>0)$ at $X=0$. 
Indeed, for the Weyl point with $\kappa_{0}<0$, we obtain the zero modes localized around the surface and the domain wall as

$$
\left[\begin{array}{l}
\psi_{1}(X) \\
\psi_{2}(X)
\end{array}\right]=\left\{\begin{array}{l}
\frac{1}{\sqrt{\lambda_{d}+\Lambda_{d}}}\left[\begin{array}{c}
\frac{+1}{\sqrt{2}} \\
\frac{+1}{\sqrt{2}}
\end{array}\right]\left\{\begin{array}{lll}
e^{+X / \lambda_{d}} & (X<0) \\
e^{-X / \Lambda_{d}} & (0<X)
\end{array} \quad\left(\kappa_{Z}>0, \delta \kappa_{Y}=0\right) \quad\right. \text { (domain wall) } \\
\frac{1}{\sqrt{\lambda_{s}+\Lambda_{s}}}\left[\begin{array}{c}
\frac{+1}{\sqrt{2}} \\
\frac{-1}{\sqrt{2}}
\end{array}\right]\left\{\begin{array}{ll}
e^{+X / \lambda_{s}} & (X<0) \\
e^{-X / \Lambda_{s}} & (0<X)
\end{array} \quad\left(\kappa_{Z}<0, \delta \kappa_{Y}=0\right) \quad \text { (surface) },\right.
\end{array}\right.
$$

where inverse penetration lengths are $\lambda_{d}^{-1}=\kappa_{Z} / \sqrt{3}>0, \Lambda_{d}^{-1}=-3|m| / 2 \kappa_{0}-\kappa_{Z} / \sqrt{3}>0, \lambda_{s}^{-1}=-3(|M|-|m|) / 2 \kappa_{0}+$ $\kappa_{Z} / \sqrt{3}>0$, and $\Lambda_{s}^{-1}=-\kappa_{Z} / \sqrt{3}>0$.

Around the Weyl points $\vec{k}_{\text {Weyl }}^{\prime}= \pm(-\sqrt{|m| / 2 t}, \sqrt{|m| / 2 t}, \sqrt{|m| / 2 t})$, by following a similar procedure used for $\hat{h}_{\Gamma \vec{k}_{\text {Weyl }}^{( \pm)}}^{(a)}$ Dirac Hamiltonian describing low-energy quasiparticle excitations is obtained as

$$
\begin{aligned}
\hat{h}_{\Gamma \vec{k}_{\mathrm{Wey} 1}^{\prime}}^{(+)}\left(-i \partial_{X}, \delta \kappa_{Y}, \kappa_{Z}\right)= & 4 t \frac{\kappa_{0}}{\sqrt{3}}\left\{-\left(\frac{\delta \kappa_{Y}}{3}-\frac{\delta \kappa_{Z}}{3}\right) \hat{\sigma}_{0}-\left(\frac{2 \delta \kappa_{Y}}{3}+\frac{\delta \kappa_{Z}}{3}\right) \hat{\sigma}_{x}\right. \\
& \left.+\frac{i \hat{\sigma}_{y}}{\sqrt{3}} \partial_{X}+\left[-\frac{\delta \kappa_{Y}}{3}+\frac{\delta \kappa_{Z}}{3}+\frac{m(X)-|m|}{4 t \kappa_{0} / \sqrt{3}}\right] \hat{\sigma}_{z}\right\},
\end{aligned}
$$

for $m(X)=+|m|$. The zero modes for the above two-component Dirac Hamiltonian are given as

$$
\left[\begin{array}{l}
\psi_{1}(X) \\
\psi_{2}(X)
\end{array}\right]=\left\{\begin{array}{l}
\frac{1}{\sqrt{\lambda_{d}+\Lambda_{d}}}\left[\begin{array}{c}
\frac{+1}{\sqrt{2}} \\
\frac{+1}{\sqrt{2}}
\end{array}\right]\left\{\begin{array}{ll}
e^{+X / \lambda_{d}} & (X<0) \\
e^{-X / \Lambda_{d}} & (0<X)
\end{array} \quad\left(\delta \kappa_{Z}<0, \delta \kappa_{Y}=0\right) \quad(\text { domain wall })\right. \\
\frac{1}{\sqrt{\lambda_{s}+\Lambda_{s}}}\left[\begin{array}{c}
\frac{+1}{\sqrt{2}} \\
\frac{+1}{\sqrt{2}}
\end{array}\right]\left\{\begin{array}{ll}
e^{+X / \lambda_{s}} & (X<0) \\
e^{-X / \Lambda_{s}} & (0<X)
\end{array} \quad\left(\delta \kappa_{Z}>0, \delta \kappa_{Y}=0\right) \quad(\text { surface }),\right.
\end{array}\right.
$$

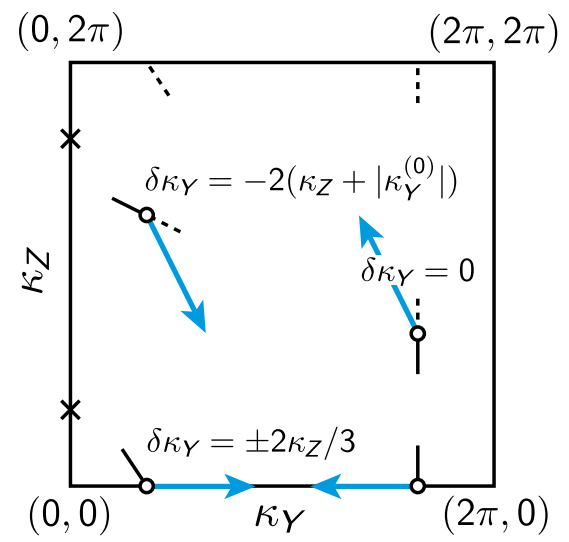

FIG. 9. Summary of solutions for the Dirac equations. Loci of the domain-wall (surface) states are represented by solid (dashed) lines, which are obtained as zero modes of the Dirac Hamiltonians $\hat{h}_{\Gamma \vec{k}_{\text {Weyl }}}^{( \pm)}$, given in Eqs. (D22)-(D25), and $\hat{h}_{\Gamma \vec{k}_{\mathrm{Wey} 1}^{\prime}}^{( \pm)}$, [see Eq. (D29)]. Open circles and crosses illustrate the Weyl points projected to the $\left(\kappa_{Y}, \kappa_{Z}\right)$ plane. where inverse penetration lengths are $\lambda_{d}^{-1}=-\delta \kappa_{Z} / \sqrt{3}>0$, $\Lambda_{d}^{-1}=-3|m| / 2 \kappa_{0}+\delta \kappa_{Z} / \sqrt{3}>0, \lambda_{s}^{-1}=-3(|M|-|m|) / 2 \kappa_{0}$ $-\delta \kappa_{Z} / \sqrt{3}>0$, and $\Lambda_{s}^{-1}=\delta \kappa_{Z} / \sqrt{3}>0$.

Similarly, we can obtain zero-mode solutions for the Dirac equations $\hat{h}_{\Gamma \vec{k}_{\text {Weyl }}^{(-)}} \vec{\psi}=E \vec{\psi}$ and $\hat{h}_{\Gamma \vec{k}_{\text {Weyl }}^{\prime}}^{(-)} \vec{\psi}=E \vec{\psi}$. These solutions are summarized in Figs. 3(b) and 9.

\section{APPENDIX E: PAIR ANNIHILATION OF WEYL ELECTRONS AT AN $L$ POINT}

To fully understand the pair annihilation of bulk Weyl points and formation of the closed loop of the Fermi surfaces on the domain walls, we need to examine the structure of the original Hamiltonian $\hat{\mathcal{K}}_{0}(\vec{k})+\hat{\mathcal{Z}}_{0}(\vec{k})$ around the $L$ point $(\pi / 4 a, \pi / 4 a, \pi / 4 a)$, where the pair annihilation occurs, beyond the applicability of the lowestorder $\vec{k} \cdot \vec{p}$ theory developed above.

Here, we expand the $8 \times 8$ Bloch Hamiltonian around the $L$ point by setting $\vec{k}=(\pi / 4 a, \pi / 4 a, \pi / 4 a)+$ $\left(\delta k_{x}, \delta k_{y}, \delta k_{z}\right)$, 


$$
\hat{\mathcal{K}}_{0}=-2 t \hat{\sigma}_{0}\left[\begin{array}{cccc}
0 & 1 & 1 & -\delta k_{z}-\delta k_{x} \\
1 & 0 & 1 & -\delta k_{y}-\delta k_{z} \\
1 & 1 & 0 & -\delta k_{x}-\delta k_{y} \\
-\delta k_{z}-\delta k_{x} & -\delta k_{y}-\delta k_{z} & -\delta k_{x}-\delta k_{y} & 0
\end{array}\right]+\mathcal{O}\left(t \delta k^{2}\right)
$$

and

$$
\hat{\mathcal{Z}}_{0}=\sqrt{2} i \zeta\left[\begin{array}{cccc}
0 & +\hat{\sigma}_{x}+\hat{\sigma}_{y} & -\hat{\sigma}_{y}-\hat{\sigma}_{z} & 0 \\
-\hat{\sigma}_{x}-\hat{\sigma}_{y} & 0 & \hat{\sigma}_{z}+\hat{\sigma}_{x} & 0 \\
\hat{\sigma}_{y}+\hat{\sigma}_{z} & -\hat{\sigma}_{z}-\hat{\sigma}_{x} & 0 & 0 \\
0 & 0 & 0 & 0
\end{array}\right]+\mathcal{O}(\zeta \delta k)
$$

It is easy to determine qualitative properties of the twocomponent Dirac equations derived from the $8 \times 8$ Hamiltonian as follows. First, we examine a level crossing at the $L$ point under the influence of $\hat{\mathcal{M}}_{0}$, which directly corresponds to the pair annihilation of the Weyl points. The level scheme at the $L$ point for $\delta \vec{k}=\overrightarrow{0}$ and $m=0$ is given by four doublets $E=-t-\sqrt{2} \zeta \pm \sqrt{9 t^{2}-6 \sqrt{2} t \zeta+6 \zeta^{2}}$, $2 t+2 \sqrt{2} \zeta$, and 0 [see Fig. 10(a)]. These doublets at the $L$

(a)
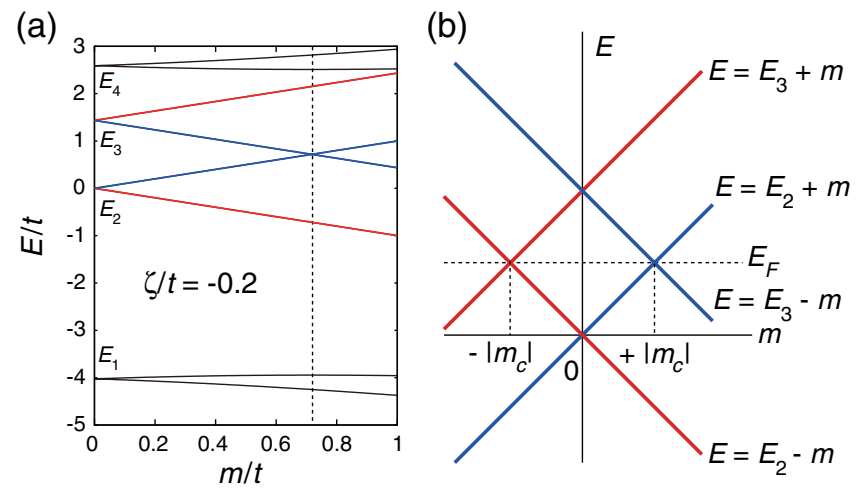

FIG. 10. Level scheme of the $L$ point and its dependence on the all-in-all-out order parameter. (a) Level splitting at the $L$ point due to the finite order parameter $m$. Here, the four doublets are located at $E_{1}=-t-\sqrt{2} \zeta-\sqrt{9 t^{2}-6 \sqrt{2} t \zeta+6 \zeta^{2}}, \quad E_{2}=0$, $E_{3}=2 t+2 \sqrt{2} \zeta$, and $E_{4}=-t-\sqrt{2} \zeta+\sqrt{9 t^{2}-6 \sqrt{2} t \zeta+6 \zeta^{2}}$, for $m=0$. The vertical dashed line shows $m=\left|m_{c}\right|$, for which the bulk Weyl points are annihilated in pairs. The two blue (red) states show the states adiabatically connected to the states that form the domain-wall state at $m=m_{c}\left(m=-m_{c}\right)$. Namely, the two blue (red) lines indicate the two solutions of the $2 \times 2$ lowenergy Dirac Hamiltonian $\hat{h}_{L}^{(+)}\left(\hat{h}_{L}^{(-)}\right)$(E7). See (b) for a more focused illustration. (b) Level splitting focused on the low-energy states, around the chemical potential. The blue states around the Fermi level $E_{F}$ form a domain-wall state confined in the side of the positive magnetization, while the red states around the Fermi level are confined in the opposite $m<0$ side of the domain wall. [See discussions below Eq. (E11).] point are classified by the irreducible representation of the point group $D_{3 d}$, as two Kramers pairs $E_{1 / 2 u}$ at $E=-t-\sqrt{2} \zeta \pm \sqrt{9 t^{2}-6 \sqrt{2} t \zeta+6 \zeta^{2}}$, one doublet $E_{3 / 2 u}$ at $E=2 t+2 \sqrt{2} \zeta$, and one Kramers pair $E_{1 / 2 g}$ at $E=0$, if we choose the site $(0,0,0)$ in Fig. 2(b) as the center of inversion, which corresponds to the seventh and eighth components of the $8 \times 8$ Bloch Hamiltonian. When we introduce a nonzero-order parameter $m$, namely, nonzero $\hat{\mathcal{M}}_{0}$, and break the time-reversal symmetry, the degeneracies of the four doublets are all lifted. Then, if $2 t+$ $2 \sqrt{2} \zeta>0$ holds, the level crossing between the two states occurs at $|m|=m_{c}$, one originally from the state at $E=$ $2 t+2 \sqrt{2} \zeta$ that splits downward for $m \neq 0$ and the other originally from the state at $E=0$ that splits upward for $m \neq 0$ [see Fig. 10(a)]. The classification of the four doublets tells us an important fact further: Although the all-in-all-out order parameter described by $\hat{\mathcal{M}}_{0}$ lifts the degeneracy of the doublets $E_{1 / 2 u}$ at $E=2 t+2 \sqrt{2} \zeta$ and the Kramers pair $E_{1 / 2 g}$ at $E=0$, the all-in-all-out order $\hat{\mathcal{M}}_{0}$ does not hybridize them. Furthermore, $\hat{\mathcal{M}}_{0}$ does not create matrix elements among $E_{1 / 2 g}$ and other doublets. Therefore, the low-energy effective theory around the pair annihilation of the Weyl points only consists of these two doublets, namely, $E_{3 / 2 u}$ and $E_{1 / 2 g}$.

Here, we construct a simplified $4 \times 4$ Hamiltonian consisting of $E_{3 / 2 u}$ and $E_{1 / 2 g}$ from a full Hamiltonian $\hat{\mathcal{K}}_{0}+\hat{\mathcal{Z}}_{0}+\hat{\mathcal{M}}_{0}$. We start with four eigenfunctions in the irreducible representations $E_{3 / 2 u}$ and $E_{1 / 2 g}$ at an $L$ point $(\pi / 4 a, \pi / 4 a, \pi / 4 a), \phi_{u \pm 3 / 2}^{T}=\left[\vec{u}_{ \pm}^{T},-\left(\hat{R} \vec{u}_{ \pm}\right)^{T},\left(\hat{R}^{2} \vec{u}_{ \pm}\right)^{T}, 0,0\right]$, and $\quad \phi_{g \pm 1 / 2}^{T}=[0,0,0,0,0,0,(1 \pm \sqrt{3})(1-i) / 2,1] /$ $\sqrt{3 \pm \sqrt{3}}$, where we introduce an $\mathrm{SU}(2)$ rotation around the $(1,1,1)$ axis $\hat{R}=\exp \left[+i(\pi / 3) \cdot(1 / 2) \cdot\left(\hat{\sigma}_{x}+\hat{\sigma}_{y}+\right.\right.$ $\left.\left.\hat{\sigma}_{z}\right) / \sqrt{3}\right]$ and define $\vec{u}_{ \pm}^{T}=(\mp \sqrt{3} / 2-i / 2)[1 \pm \sqrt{3}$, $1-i] / \sqrt{6 \pm 2 \sqrt{3}}$. Then, the $4 \times 4$ Hamiltonian up to the $\mathcal{O}(t \delta k)$ and $\mathcal{O}(t \zeta)$ is given as follows with a new 
momentum frame $k_{1}=\left(\delta k_{x}+\delta k_{y}-2 \delta k_{z}\right) / \sqrt{6}, \quad k_{2}=$ $\left(-\delta k_{x}+\delta k_{y}\right) / \sqrt{2}$, and $k_{3}=\left(\delta k_{x}+\delta k_{y}+\delta k_{z}\right) / \sqrt{3}$

$$
\left[\begin{array}{cccc}
E_{g}-m & 0 & +\frac{2}{\sqrt{6}} t k_{e}^{*} & +\frac{2}{\sqrt{3}} t k_{e}^{*} \\
0 & E_{g}+m & -\frac{2}{\sqrt{3}} t k_{e} & +\frac{2}{\sqrt{6}} t k_{e} \\
+\frac{2}{\sqrt{6}} t k_{e} & -\frac{2}{\sqrt{3}} t k_{e}^{*} & E_{u}-m & 0 \\
+\frac{2}{\sqrt{3}} t k_{e} & +\frac{2}{\sqrt{6}} t k_{e}^{*} & 0 & E_{u}+m
\end{array}\right],
$$

where $E_{g}=0, E_{u}=2 t+2 \sqrt{2} \zeta$, and $k_{e}=k_{1}-i k_{2}$.

By introducing external gauge fields to the $4 \times 4$ Hamiltonian (E3), we can easily construct Landau levels and clarify its topological natures, such as manifestation of the chiral anomaly. An orbital part of external magnetic fields $B$ is introduced through introducing a real-space coordinate $x_{1}$ corresponding to $k_{1}$ as $k_{1} \rightarrow-i \partial_{x_{1}}, k_{2} \rightarrow$ $k_{2}-e B x_{1}$. The $4 \times 4$ Hamiltonian (E3) is rewritten as

$$
\left[\begin{array}{cccc}
E_{g}-m & 0 & -2 i t \sqrt{\frac{e B}{3}} \hat{\varphi}^{-} & -2 i t \sqrt{\frac{2 e B}{3}} \hat{\varphi}^{-} \\
0 & E_{g}+m & -2 i t \sqrt{\frac{2 e B}{3}} \hat{\ell}^{+} & +2 i t \sqrt{\frac{e B}{3}} \hat{\ell}^{+} \\
+2 i t \sqrt{\frac{e B}{3}} \hat{\ell}^{+}+2 i t \sqrt{\frac{2 e B}{3}} \hat{\ell}^{-} & E_{u}-m & 0 \\
+2 i t \sqrt{\frac{2 e B}{3}} \hat{\ell}^{+} & -2 i t \sqrt{\frac{e B}{3}} \hat{\ell}^{-} & 0 & E_{u}+m
\end{array}\right],
$$

where ladder operators $\hat{\ell}^{-}=+\partial_{x_{1}} / \sqrt{2 e B}+\sqrt{e B / 2}\left(x_{1}-\right.$ $\left.k_{2} / e B\right)$ and $\hat{\ell}^{+}=-\partial_{x_{1}} / \sqrt{2 e B}+\sqrt{e B / 2}\left(x_{1}-k_{2} / e B\right)$ are introduced. By using orthonormalized eigenfunctions of harmonic oscillators $\varphi_{n}(x)$ satisfying $\hat{\ell}^{-} \varphi_{n}\left(x_{1}-k_{2} / e B\right)=$ $\sqrt{n} \varphi_{n-1}\left(x_{1}-k_{2} / e B\right), \quad \hat{\ell}^{+} \varphi_{n}\left(x_{1}-k_{2} / e B\right)=\sqrt{n+1} \varphi_{n+1}$ $\left(x_{1}-k_{2} / e B\right)$, and $\int d x \varphi_{n}^{*}(x) \varphi_{n^{\prime}}(x)=\delta_{n, n^{\prime}}$, we obtain eigenvectors of Eq. (E4) for Landau levels.

Two Landau levels become important when topological properties of the magnetic domain walls are discussed: The first one is an eigenvector $\left[0, \varphi_{0}\left(x_{1}-k_{2} / e B\right), 0,0\right]^{T}$ with an eigenvalue $E_{g}+m$. The other is given by an eigenvector $\left[0, b_{1} \varphi_{1}\left(x_{1}-k_{2} / e B\right), b_{2} \varphi_{0}\left(x_{1}-k_{2} / e B\right), c \varphi_{0}\left(x_{1}-k_{2} / e B\right)\right]^{T}$, where $b_{1}, b_{2} \rightarrow 0$ and $c \rightarrow 1$ for $|m / t| \gg 1$ with an eigenvalue approaching $E_{u}+m$. These two states are nothing but a manifestation of the chiral anomaly; in other words, the zeroth Landau levels of Weyl nodes are annihilated in pairs for $E_{g}+m=E_{u}-m$ or $E_{g}-m=$ $E_{u}+m$ at the $L$ points. The asymptotic behavior of these two zeroth Landau levels is also captured by decoupling the $4 \times 4$ Hamiltonian (E4) into a set of $2 \times 2$ effective Hamiltonians $\hat{h}_{L}^{( \pm)}$.

These $2 \times 2$ effective Hamiltonians $\hat{h}_{L}^{(+)}$and $\hat{h}_{L}^{(-)}$consist of the third and second, and, the first and fourth components of the $4 \times 4$ Hamiltonian (E4), respectively, as

$$
\begin{aligned}
\hat{h}_{L}^{(+)} & =\left[\begin{array}{cc}
E_{u}-m & -\frac{2}{\sqrt{3}} t k_{e}^{*} \\
-\frac{2}{\sqrt{3}} t k_{e} & E_{g}+m
\end{array}\right]-\frac{E_{u}+E_{g}}{2} \hat{\sigma}_{0}, \\
& =\left(\frac{E_{u}-E_{g}}{2}-m\right) \hat{\sigma}_{z}-\frac{2}{\sqrt{3}} t k_{1} \hat{\sigma}_{x}+\frac{2}{\sqrt{3}} t k_{2} \hat{\sigma}_{y}
\end{aligned}
$$

and

$$
\begin{aligned}
\hat{h}_{L}^{(-)} & =\left[\begin{array}{cc}
E_{g}-m & +\frac{2}{\sqrt{3}} t k_{e}^{*} \\
+\frac{2}{\sqrt{3}} t k_{e} & E_{u}+m
\end{array}\right]-\frac{E_{u}+E_{g}}{2} \hat{\sigma}_{0}, \\
& =\left(\frac{E_{g}-E_{u}}{2}-m\right) \hat{\sigma}_{z}+\frac{2}{\sqrt{3}} t k_{1} \hat{\sigma}_{x}-\frac{2}{\sqrt{3}} t k_{2} \hat{\sigma}_{y},
\end{aligned}
$$

after subtracting the common diagonal term $\left(E_{u}+E_{q}\right) \hat{\sigma}_{0} / 2$. In the following, we further derive onedimensional Dirac equations based on $\hat{h}_{L}^{(+)}$and $\hat{h}_{L}^{(-)}$. The three-dimensional Weyl equations introduced in Sec. V are also obtained as follows. First, we apply a unitary transformation $\left(\hat{\sigma}_{x}, \hat{\sigma}_{y}, \hat{\sigma}_{z}\right) \rightarrow\left(-\hat{\sigma}_{x},+\hat{\sigma}_{y},-\hat{\sigma}_{z}\right)$ for $\hat{h}_{L}^{(+)}$and $\left(\hat{\sigma}_{x}, \hat{\sigma}_{y}, \hat{\sigma}_{z}\right) \rightarrow\left(+\hat{\sigma}_{x},-\hat{\sigma}_{y},-\hat{\sigma}_{z}\right)$ for $\hat{h}_{L}^{(-)}$. Second, we rescale $\left(2 t k_{1} / \sqrt{3}, 2 t k_{2} / \sqrt{3}, v k_{3}\right)$ to $\left(p_{1}, p_{2}, p_{3}\right)$ with a constant $v$ and introduce higher-order terms proportional to $p_{3}^{2}$ to reproduce pairwise annihilation of the Weyl nodes.

Now, we focus on the $(01 \overline{1})$ domain wall for illustrative purposes and note that, up to linear orders in $\kappa_{X}, \delta \kappa_{Y}$, and $\kappa_{Z}, 2 \times 2$ Dirac Hamiltonians describing low-energy physics do not contain terms proportional to $\delta \kappa_{Y}$, due to the point symmetry of the electronic band around $\vec{k}=(\pi / 4 a, \pi / 4 a, \pi / 4 a)$. Here, we introduce a new oblique coordinate $\left(\kappa_{X}, \delta \kappa_{Y}, \kappa_{Z}\right)$ through $\left(\delta k_{x}, \delta k_{y}, \delta k_{z}\right)=$ $\left(-\delta \kappa_{Y}-\kappa_{Z},+\kappa_{X}-\delta \kappa_{Y},-\kappa_{X}-\delta \kappa_{Y}\right)$ for the (011) domain wall. It is easy to see that, along the (111) direction, the band dispersion shows a quadratic band crossing at the pair annihilation of the Weyl points. In other words, along the $\kappa_{Y}$ axis parallel to the (111) direction, the linear dispersion disappears. Therefore, in general, the pair of the low-energy $2 \times 2$ Dirac Hamiltonians around the $L$ point is given as

$$
\begin{aligned}
\hat{h}_{L}^{( \pm)}= & -\left[m \mp\left|m_{c}\right|\right] \hat{\sigma}_{z}+\left(v_{X x}^{( \pm)} \hat{\sigma}_{x}+v_{X y}^{( \pm)} \hat{\sigma}_{y}\right) \kappa_{X} \\
& +\left(v_{Z x}^{( \pm)} \hat{\sigma}_{x}+v_{Z y}^{( \pm)} \hat{\sigma}_{y}\right) \kappa_{Z},
\end{aligned}
$$

where velocities $v_{X x}^{( \pm)}=\mp \sqrt{2} t, v_{X y}^{( \pm)}= \pm 2 t / \sqrt{6}, v_{Z x}^{( \pm)}=$ $\pm \sqrt{2} t / 3$, and $v_{Z y}^{( \pm)}= \pm 2 t / \sqrt{6}$ are introduced, and $\left|m_{c}\right|=$ $t+\sqrt{2} \zeta$ is the critical value of $m$ for the pair annihilation of the Weyl points. Here, we emphasize that the above set of the Dirac Hamiltonian exploits the low-energy Hilbert space $E_{1 / 2 u} \oplus E_{1 / 2 g}$. The solution of Eq. (E7) as a function 
of $m$ is illustrated in Fig. 10(b), which has, of course, the same structure as Fig. 10(a) in the low-energy region. By replacing $m$ and $\kappa$ with $m(X)$ and $-i \partial_{X}$, respectively, we obtain the following 1D Dirac equation:

$$
\begin{aligned}
\hat{h}_{L}^{( \pm)}(X)= & -\left[m(X) \mp\left|m_{c}\right|\right] \hat{\sigma}_{z}-i \tilde{v}_{X}^{( \pm)}\left(\cos \varphi_{X}^{( \pm)} \hat{\sigma}_{x}\right. \\
& \left.+\sin \varphi_{X}^{( \pm)} \hat{\sigma}_{y}\right) \partial_{X}+\tilde{v}_{Z}^{( \pm)}\left(\cos \varphi_{Z}^{( \pm)} \hat{\sigma}_{x}\right. \\
& \left.+\sin \varphi_{Z}^{( \pm)} \hat{\sigma}_{y}\right) \kappa_{Z},
\end{aligned}
$$

where velocities $\tilde{v}_{X}^{( \pm)}$and $\tilde{v}_{Z}^{( \pm)}$and phases $\varphi_{X}^{( \pm)}$and $\varphi_{Z}^{( \pm)}$are defined through $\tilde{v}_{X}^{( \pm)} e^{i \varphi_{X}^{( \pm)}}=v_{X x}^{( \pm)}+i v_{X y}^{( \pm)}$and $\tilde{v}_{Z}^{( \pm)} e^{i \varphi_{Z}^{( \pm)}}=$ $v_{Z x}^{( \pm)}+i v_{Z y}^{( \pm)}$. Here, the $(01 \overline{1})$ domain wall with an all-out domain for $X<0$ and an all-in domain for $X>0$ is described by the following $X$-dependent mass term

$$
m(X)=+|m| \theta(-X)-|m| \theta(+X)
$$

which is justified from the level scheme splitting illustrated in Fig. 10(b).

Then, the solution for the 1D Dirac equation $\hat{h}_{L}^{(+)}(X)$, after the pair annihilation of the Weyl points $\left(|m|>\left|m_{c}\right|\right)$

$$
\hat{h}_{L}^{(+)}(X) \vec{\psi}=0 \cdot \vec{\psi}
$$

is given by

$$
\begin{aligned}
\vec{\psi}(X)= & \frac{1}{\sqrt{\lambda_{d L}+\Lambda_{d L}}}\left[\theta(-X) e^{+X / \lambda_{d L}}\right. \\
& +\theta(+X) e^{\left.-X / \Lambda_{d L}\right]}\left[\begin{array}{c}
1 / \sqrt{2} \\
i e^{i \varphi_{X}} / \sqrt{2}
\end{array}\right]
\end{aligned}
$$

where the inverse penetration lengths are $\lambda_{d L}^{-1}=$ $\left(|m|-\left|m_{c}\right|\right) / \tilde{v}_{X}^{(+)}>0$ and $\Lambda_{d L}^{-1}=|m| / \tilde{v}_{X}^{(+)}>0$, along the locus defined by $\kappa_{Z}=0$. The above solution is mainly confined in the side of the positive magnetization $m>0$. By solving the other 1D Dirac equation $\hat{h}_{L}^{(-)}(X) \vec{\psi}=0 \cdot \vec{\psi}$, the domain-wall state confined in the side of the negative magnetization $m<0$ is obtained in the same manner.

We note that, for $\kappa_{Z}=0$, an operator

$$
\hat{\Gamma}=-\sin \varphi_{X}^{(+)} \hat{\sigma}_{x}+\cos \varphi_{X}^{(+)} \hat{\sigma}_{y}
$$

becomes a chiral one for the above 1D Dirac equation that satisfies the following identities:

$$
\hat{\Gamma}^{\dagger} \hat{h}_{L}^{(+)}(X) \hat{\Gamma}=-\hat{h}_{L}^{(+)}(X)
$$

and $\hat{\Gamma}^{\dagger} \hat{\Gamma}=1$. Here, we also note that, in the above derivation, the chemical potential is assumed to be pinned at the Weyl points.
Therefore, the domain-wall zero modes given by the solution to Eq. (E11) are protected by the chiral operator $\hat{\Gamma}$, in other words, protected by the chiral symmetry of $\hat{h}_{L}^{(+)}(X)$ with $\kappa_{Z}=0$ (see Ref. [2]). The classification of the topological insulators introduced in Ref. [2] tells that the 1D chiral Dirac equations derived for the low-energy physics around the $L$ point describe the AIII Chern insulators. From the above derivation, our 1D Dirac equations turn out to be chiral, at least, around the $L$ point. As long as the chemical potential is pinned at the Weyl point or the center of the bulk gap, then the zero modes are preserved.

\section{APPENDIX F: UNRESTRICTED HARTREE-FOCK TREATMENT}

We use the following mean-field decoupling throughout the present paper for the unrestricted Hartree-Fock approximation:

$$
\begin{aligned}
\hat{n}_{i \uparrow} \hat{n}_{i \downarrow} \simeq & {\left[\hat{c}_{i \uparrow}^{\dagger}, \hat{c}_{i \downarrow}^{\dagger}\right]\left(\frac{\rho_{i}}{2} \hat{\sigma}_{0}-\frac{\vec{\mu}_{i} \cdot \overrightarrow{\hat{\sigma}}}{2}\right)\left[\begin{array}{c}
\hat{c}_{i \uparrow} \\
\hat{c}_{i \downarrow}
\end{array}\right] } \\
& -\left\langle\hat{n}_{i \uparrow}\right\rangle\left\langle\hat{n}_{i \downarrow}\right\rangle+\left\langle\hat{c}_{i \uparrow}^{\dagger} \hat{c}_{i \downarrow}\right\rangle\left\langle\hat{c}_{i \downarrow}^{\dagger} \hat{c}_{i \uparrow}\right\rangle,
\end{aligned}
$$

where the mean fields are defined as

$$
\begin{gathered}
\rho_{i}=\left\langle\hat{n}_{i \uparrow}\right\rangle+\left\langle\hat{n}_{i \downarrow}\right\rangle, \\
\mu_{i}^{x}=\left\langle\hat{c}_{i \uparrow}^{\dagger} \hat{c}_{i \downarrow}\right\rangle+\left\langle\hat{c}_{i \downarrow}^{\dagger} \hat{c}_{i \uparrow}\right\rangle, \\
\mu_{i}^{y}=-i\left\langle\hat{c}_{i \uparrow}^{\dagger} \hat{c}_{i \downarrow}\right\rangle+i\left\langle\hat{c}_{i \downarrow}^{\dagger} \hat{c}_{i \uparrow}\right\rangle, \\
\mu_{i}^{z}=\left\langle\hat{n}_{i \uparrow}\right\rangle-\left\langle\hat{n}_{i \downarrow}\right\rangle .
\end{gathered}
$$

For instance, the all-in-all-out order is described with the spin components of the mean fields $\vec{\mu}_{i}$ pointing in the configuration of all-in and all-out directions [18]. Here, a bracket $\langle\hat{O}\rangle$ means the self-consistent average of a singleparticle operator $\hat{O}$.

\section{APPENDIX G: SUPERCELLS}

For fully unrestricted Hartree-Fock calculations, we use supercells to describe domain walls.

For the $(01 \overline{1})$ domain-wall calculations, we specify the sites within the supercell as

$$
\vec{r}_{n \ell L}=\vec{r}_{n}+\ell\left[\begin{array}{c}
-2 a \\
+2 a \\
+0
\end{array}\right]+L\left[\begin{array}{c}
0 \\
+2 a \\
-2 a
\end{array}\right]
$$

where $n, \ell$, and $L$ are integers, and $\vec{r}_{n}(n=1,2,3,4)$ is the location of the $n$th site in the unit cell: $\vec{r}_{1}=(a, 0, a)^{T}$, $\vec{r}_{2}=(0, a, a)^{T}, \vec{r}_{3}=(a, a, 0)^{T}$, and $\vec{r}_{4}=(0,0,0)^{T}$. 
For the (111) domain wall,

$$
\vec{r}_{n 0 L}=\vec{r}_{n}+L\left[\begin{array}{c}
-2 a \\
0 \\
-2 a
\end{array}\right]
$$

and, for the (100) domain wall,

$$
\vec{r}_{n \ell L}=\vec{r}_{n}+\ell\left[\begin{array}{c}
-2 a \\
+2 a \\
+0
\end{array}\right]+L\left[\begin{array}{c}
+2 a \\
+2 a \\
0
\end{array}\right] .
$$

For actual unrestricted Hartree-Fock calculations, we chose $\ell=0,1$ and $L=0,1, \ldots, 39$. We make sharp domain walls between $L=19$ and $L=20$ as the initial conditions. We use periodic boundary conditions parallel to the domain walls and open boundary conditions perpendicular to the domain walls.

[1] M.Z. Hasan and C. L. Kane, Colloquium: Topological Insulators, Rev. Mod. Phys. 82, 3045 (2010).

[2] S. Ryu, A. P. Schnyder, A. Furusaki, and A. W. W. Ludwig, Topological Insulators and Superconductors: Tenfold Way and Dimensional Hierarchy, New J. Phys. 12, 065010 (2010).

[3] B. A. Volkov and O.A. Pankratov, Two-Dimensional Massless Electrons in an Inverted Contact, JETP Lett. 42, 178 (1985) [Pis'ma Zh. Eksp. Teor. Fiz. 42, 145 (1985)].

[4] S. V. Pankratov, S. V. Pakhomov, and B. A. Volkov, Supersymmetry in Heterojunctions: Band-Inverting Contact on the Basis of $\mathrm{Pb}_{1-x} \mathrm{Sn}_{x} \mathrm{Te}$ and $\mathrm{Hg}_{1-x} \mathrm{Cd}_{x} \mathrm{Te}$, Solid State Commun. 61, 93 (1987).

[5] E. Fradkin, E. Dagotto, and D. Boyanovsky, Physical Realization of the Parity Anomaly in Condensed Matter Physics, Phys. Rev. Lett. 57, 2967 (1986).

[6] X. Wan, A. M. Turner, A. Vishwanath, and S. Y. Savrasov, Topological Semimetal and Fermi-Arc Surface States in the Electronic Structure of Pyrochlore Iridates, Phys. Rev. B 83, 205101 (2011).

[7] A. H. C. Neto, F. Guinea, N. M. R. Peres, K. S. Novoselov, and A. K. Geim, The Electronic Properties of Graphene, Rev. Mod. Phys. 81, 109 (2009).

[8] K. Sun, H. Yan, E. Fradkin, and S. A. Kivelson, Topological Insulators and Nematic Phases from Spontaneous Symmetry Breaking in 2D Fermi Systems with a Quadratic Band Crossing, Phys. Rev. Lett. 103, 046811 (2009).

[9] D. Yanagishima and Y. Maeno, Metal-Nonmetal Changeover in Pyrochlore Iridates, J. Phys. Soc. Jpn. 70, 2880 (2001).

[10] K. Matsuhira, M. Wakeshima, Y. Hinatsu, and S. Takagi, Metal-Insulator Transitions in Pyrochlore Oxides $\mathrm{Ln}_{2} \mathrm{Ir}_{2} \mathrm{O}_{7}$, J. Phys. Soc. Jpn. 80, 094701 (2011).

[11] K. Tomiyasu, K. Matsuhira, K. Iwasa, M. Watahiki, S. Takagi, M. Wakeshima, Y. Hinatsu, M. Yokoyama, K. Ohoyama, and K. Yamada, Emergence of Magnetic Long-Range Order in Frustrated Pyrochlore $\mathrm{Nd}_{2} \mathrm{Ir}_{2} \mathrm{O}_{7}$ with
Metal-Insulator Transition, J. Phys. Soc. Jpn. 81, 034709 (2012).

[12] K. Ueda, J. Fujioka, Y. Takahashi, T. Suzuki, S. Ishiwata, Y. Taguchi, and Y. Tokura, Variation of Charge Dynamics in the Course of Metal-Insulator Transition for Pyrochlore-Type $\mathrm{Nd}_{2} \mathrm{Ir}_{2} \mathrm{O}_{7}$, Phys. Rev. Lett. 109, 136402 (2012).

[13] J. J. Ishikawa, E. C. T. O'Farrell, and S. Nakatsuji, Continuous Transition between Antiferromagnetic Insulator and Paramagnetic Metal in the Pyrochlore Iridate $\mathrm{Eu}_{2} \mathrm{Ir}_{2} \mathrm{O}_{7}$, Phys. Rev. B 85, 245109 (2012).

[14] K. Matsuhira, M. Tokunaga, M. Wakeshima, Y. Hinatsu, and S. Takagi, Giant Magnetoresistance Effect in the MetalInsulator Transition of Pyrochlore Oxide $\mathrm{Nd}_{2} \mathrm{Ir}_{2} \mathrm{O}_{7}$, J. Phys. Soc. Jpn. 82, 023706 (2013).

[15] C. Kittel, Introduction to Solid State Physics (Wiley, New York, 2005).

[16] S.S.P. Parkin, in Spin Dependent Transport in Magnetic Nanostructures, edited by S. Maekawa and T. Shinjo (CRC Press, Boca Raton, Florida, 2002), Chap. 5, p. 237.

[17] W. Witczak-Krempa, G. Chen, Y.-B. Kim, and L. Balents, Correlated Quantum Phenomena in the Strong Spin-Orbit Regime, Annu. Rev. Condens. Matter Phys. 5, 57 (2014).

[18] W. Witczak-Krempa and Y.-B. Kim, Topological and Magnetic Phases of Interacting Electrons in the Pyrochlore Iridates, Phys. Rev. B 85, 045124 (2012).

[19] S. M. Disseler, S. R. Giblin, C. Dhital, K. C. Lukas, S. D. Wilson, and M. J. Graf, Magnetization and Hall Effect Studies on the Pyrochlore Iridate $\mathrm{Nd}_{2} \mathrm{Ir}_{2} \mathrm{O}_{7}$, Phys. Rev. B 87, 060403(R) (2013).

[20] A. A. Abrikosov and S. D. Beneslavskii, Possible Existence of Substances Intermediate between Metals and Dielectrics, Sov. Phys. JETP 32, 699 (1971) [Zh. Eksp. Teor. Fiz. 59, 1280 (1970)].

[21] E. M. Lifshitz and L. P. Pitaevskii, Statistical Physics, Part 2 (Oxford University Press, New York, 2002).

[22] E.-G. Moon, C. Xu, Y.-B. Kim, and L. Balents, Non-Fermi Liquid and Topological States with Strong Spin-Orbit Coupling, Phys. Rev. Lett. 111, 206401 (2013).

[23] D. Pesin and L. Balents, Mott Physics and Hand Topology in Materials with Strong Spin-Orbit Interaction, Nat. Phys. 6, 376 (2010).

[24] A. Go, W. Witczak-Krempa, G. S. Jeon, K. Park, and Y.-B. Kim, Correlation Effects on 3D Topological Phases: From Bulk to Boundary, Phys. Rev. Lett. 109, 066401 (2012).

[25] H.-M. Guo and M. Franz, Three-Dimensional Topological Insulators on the Pyrochlore Lattice, Phys. Rev. Lett. 103, 206805 (2009).

[26] M. Kurita, Y. Yamaji, and M. Imada, Topological Insulators from Spontaneous Symmetry Breaking Induced by Electron Correlation on Pyrochlore Lattices, J. Phys. Soc. Jpn. 80, 044708 (2011).

[27] M. Elhajal, B. Canals, R. Sunyer, and C. Lacroix, Ordering in the Pyrochlore Antiferromagnet due to Dzyaloshinsky-Moriya Interactions, Phys. Rev. B 71, 094420 (2005). 
[28] J. M. Luttinger, Quantum Theory of Cyclotron Resonance in Semiconductors: General Theory, Phys. Rev. 102, 1030 (1956).

[29] S. Murakami, N. Nagaosa, and S.-C. Zhang, SU(2) Non-Abelian Holonomy and Dissipationless Spin Current in Semiconductors, Phys. Rev. B 69, 235206 (2004).

[30] M. S. Dresselhaus, G. Dresselhaus, and A. Jorio, Group Theory-Application to the Physics of Condensed Matter (Springer-Verlag, Berlin, 2008).

[31] M. A. Silaev and G.E. Volovik, Topological Fermi Arcs in Superfluid ${ }^{3} \mathrm{He}$, Phys. Rev. B 86, 214511 (2012).

[32] K.-Y. Yang, Y.-M. Lu, and Y. Ran, Quantum Hall Effects in a Weyl Semimetal: Possible Application in Pyrochlore Iridates, Phys. Rev. B 84, 075129 (2011).

[33] R. Jackiw and C. Rebbi, Solitons with Fermion Number 1/2, Phys. Rev. D 13, 3398 (1976).

[34] F. Zhang, C. L. Kane, and E. J. Mele, Surface States of Topological Insulators, Phys. Rev. B 86, 081303(R) (2012).

[35] H. B. Nielsen and M. Ninomiya, The Adler-Bell-Jackiw Anomaly and Weyl Fermions in a Crystal, Phys. Lett. 130B, 389 (1983).
[36] W. Yao, S. A. Yang, and Q. Niu, Edge States in Graphene: From Gapped Flat-Band to Gapless Chiral Modes, Phys. Rev. Lett. 102, 096801 (2009).

[37] J. C. Y. Teo and C. L. Kane, Topological Defects and Gapless Modes in Insulators and Superconductors, Phys. Rev. B 82, 115120 (2010).

[38] T. Kawarabayashi, Y. Hatsugai, T. Morimoto, and H. Aoki, Generalized Chiral Symmetry and Stability of Zero Modes for Tilted Dirac Cones, Phys. Rev. B 83, 153414 (2011).

[39] A. J. Heeger, S. Kivelson, J. R. Schrieffer, and W.-P. Su, Solitons in Conducting Polymers, Rev. Mod. Phys. 60, 781 (1988).

[40] T. Arima, Time-Reversal Symmetry Breaking and Consequent Physical Responses Induced by All-In/All-Out Type Magnetic Order on the Pyrochlore Lattice, J. Phys. Soc. Jpn. 82, 013705 (2013).

[41] A. Kitaev, in Proceedings of the L. D. Landau Memorial Conference "Advances in Theoretical Physics," Chernogolovka, Russia, 2008 (American Institute of Physics, Melville, NY, 2009); arXiv:0901.2686.

[42] X.-G. Wen, Symmetry-Protected Topological Phases in Noninteracting Fermion Systems, Phys. Rev. B 85, 085103 (2012). 